\title{
The Navier-Stokes-Vlasov-Fokker-Planck System as a Scaling Limit of Particles in a Fluid
}

\author{
Franco Flandoli(i), Marta Leocata and Cristiano Ricci \\ Communicated by G. P. Galdi
}

\begin{abstract}
Convergence of a system of particles, interacting with a fluid, to Navier-Stokes-Vlasov-Fokker-Planck system is studied. The interaction between particles and fluid is described by Stokes drag force. The empirical measure of particles is proved to converge to the Vlasov-Fokker-Planck component of the system and the velocity of the fluid coupled with the particles converges in the uniform topology to the the Navier-Stokes component. A new uniqueness result for the PDE system is added.
\end{abstract}

Keywords. Particle-system, Mean-Field, scaling limits, Vlasov-Navier-Stokes, Kinetic theory.

\section{Introduction}

In the theory of multiphase flows, the coupled PDE system called Navier-Stokes-Vlasov-Fokker-Planck is a way of modeling the behavior of a large number of particles immersed into a fluid. It is made by two major components: a vector field $u$, representing the velocity of the fluid at a given time and position, and a scalar valued function $F$, representing the density on phase space of the particles immersed in the fluid. In the incompressible case, when the interaction between particles and fluid is modelled by Stokes drag force, the system is given by the following equations

$$
\left\{\begin{array}{l}
\partial_{t} u=\Delta u-u \cdot \nabla u-\nabla \pi-\int_{\mathbb{R}^{d}}(u-v) F d v \\
\operatorname{div}(u)=0 \\
\partial_{t} F+v \cdot \nabla_{x} F+\operatorname{div}_{v}((u-v) F)=\frac{\sigma^{2}}{2} \Delta_{v} F .
\end{array}\right.
$$

Often the case $\sigma=0$ is considered in the literature. Here we deal with the case $\sigma>0$ because of technical reasons. The case $\sigma=0$ is usually called Vlasov-Navier-Stokes (VNS); the case $\sigma>0$, NavierStokes-Vlasov-Fokker-Planck. In the sequel, for simplicity of notations, we will often call VNS also the system above with $\sigma>0$.

The PDE description for the density of particles is reasonable when the number of particles is very large and overcomes the problem of describing the details of each single particle. The aim of this paper is to prove that this simplification is correct: we prove that a system composed by Newtonian particles and fluid converges to the PDE system when the number of particles tends to infinity.

The mathematical analysis of the coupled system (1) in dimension $d=2,3$ has received much attention in the past years. A first result of global existence of weak solutions and large asymptotic for StokesVlasov system in a bounded domain appeared in [20]. Existence of weak solutions has been extended to the Navier-Stokes case, hence including the convection term in the equation for the fluid, in a periodic domain in [5]. Global existence of smooth solutions with small data for Navier-Stokes-Vlasov-FokkerPlanck was obtained first in [17]. In [27] global existence for smooth solutions is generalized for large data. Recent results on the topic of uniqueness have been obtained in the case $\sigma=0$ in [21]. We shall prove a variant of these results adapted to the regularity of our solutions. Uniqueness plays a fundamental role 
in the mathematical problem we are interested in; existence is less relevant because it is obtained as a byproduct of our convergence result.

As said above, the aim of this work is to investigate a coupling between the fluid and a particle system, which converges, in the limit of large number of particles, to system (1). The literature on this topic is still fragmentary. The works $[18,19]$, present results of PDE to PDE convergence, only implicitly motivated by particle arguments. The works $[1-3,9-11,22]$ aim to treat links between particles and fluid but, in the trade-off between different levels of mathematical complexity and physical realism: there in a simplified fluid regime, the correct boundary condition for the interaction between finite size particle and the fluid is included. Compared to these works, our choice here is a sort of phenomenological description of interaction between particles and fluid, that keeps the structure of Stokes drag force and that maintains the usual Navier-Stokes regime. Our attention is devoted to others technical problems related to the macroscopic limit, instead of the very difficult problem of the precise boundary conditions between particles and fluid. The microscopic system considered here has the form

$$
\left\{\begin{array}{l}
\frac{\partial u^{N}}{\partial t}=\Delta u^{N}-u^{N} \cdot \nabla u^{N}-\nabla \pi^{N}-\frac{1}{N} \sum_{i=1}^{N}\left(u_{\varepsilon_{N}}^{N}\left(t, X_{t}^{i, N}\right)-V_{t}^{i}\right) \delta_{X_{t}^{i, N}}^{\varepsilon_{N}}, \\
\operatorname{div}\left(u^{N}\right)=0 \\
\left\{\begin{array}{l}
d X_{t}^{i, N}=V_{t}^{i, N} d t \\
d V_{t}^{i, N}=\left(u_{\varepsilon_{N}}^{N}\left(t, X_{t}^{i, N}\right)-V_{t}^{i, N}\right) d t+\sigma d B_{t}^{i}
\end{array}\right.
\end{array}\right.
$$

where $N$ is the number of particles and $\left(X_{t}^{i, N}, V_{t}^{i, N}\right)$ are position and velocity of the particles. The equations for the fluid velocity and pressure $\left(u^{N}, \pi^{N}\right)$ are given by the classical Navier-Stokes equations for an incompressible Newtonian fluid with an interaction with particles of discrete type. We choose a phenomenological description of the interaction:

i) the intensity of the force exerted by the fluid on each single particle is given by the difference between the particle velocity and a local average of fluid velocity around particle position

$$
u_{\varepsilon_{N}}^{N}\left(t, X_{t}^{i, N}\right)=\left(\theta^{0, \varepsilon_{N}} * u_{t}^{N}\right)\left(X_{t}^{i, N}\right) ;
$$

ii) viceversa the force exerted by each single particle on the fluid is given by Newton's third law: the intensity of the force is the same oh (i), but with the opposite sign. Moreover we impose an action distributed in a small neighbor of particle position, as described by the mollified delta Dirac function

$$
\delta_{X_{t}^{i, N}}^{\varepsilon_{N}}(x)=\theta^{0, \varepsilon_{N}}\left(x-X_{t}^{i, N}\right) .
$$

The choice to use local averages and locally distributed action is obviously an artefact, convenient for the mathematical investigation; still it preserves the idea that particles are not just points but finite objects, or at least objects with a finite action radius, a sort of small boundary layer of interaction with the fluid.

Finally, let us comment on our previous works $[13,14]$. They both deal with a similar particle system coupled with the fluid and the question of its scaling limit. However, they are affected by important restrictions. The paper [13] discusses only the so called two steps approach. In this setting one keeps $\varepsilon$ fixed when $N \rightarrow \infty$ and removes $\epsilon$ only later, as a second step. As usual, the analysis of such disjoint limits is much simpler: the first step is a classical mean field problem (opposite to the problem considered here, see the next section on the technical difficulties), the second step is a question of convergence of PDEs to PDEs (essentially a repetition of schemes known from the proofs of existence theorems for the limit system). One can mix the parameters a posteriori, taking subsequences, but the conditions on the link are quite unrealistic and restrictive. As in the present work, the paper [14] treats the joint limit in the two parameters, but a special bounded modification of Stokes law is required and due to lack of a suitable uniqueness result, we prove only convergence of subsequences. Compared to [13,14], the result proved here is complete, without the main restrictions of those works. For future research, however, it would be interesting to extend further the range of the parameter $\beta$ that quantifies the radius of interaction between a particle and the surrounding 
fluid. See below and in the same vein how to treat more realistic boundary conditions between particles and fluid.

\subsection{Difficulties}

In this subsection we aim to highlight the difficulties we met in proving the convergence from the discrete to the continuous model. Apparently it looks a mean field result but several aspects are far from standard, as we now describe.

1.1.1. Uniform Control on Velocity and Vorticity Creation by Particles. The rough structure of the particle approximation used here is of a mean field type. The empirical measure $S_{t}^{N}$ of the particles

$$
S_{t}^{N}=\frac{1}{N} \sum_{i=1}^{N} \delta_{\left(X_{t}^{i, N}, V_{t}^{i, N}\right)}
$$

(see also Sect. 2) will be proved to converge to the solution $F_{t}(x, v)$ of the Vlasov component of our system (in parallel, the approximation of the velocity field will converge to the limit velocity field). However in classical mean field problems, first it is proved that $S_{t}^{N}$ converges to $F_{t}(x, v)$ in the weak sense of measures, then one can pass to the limit, thanks to the non-local structure of the nonlinear terms. In our problem, there is a main difficulty: $S_{t}^{N}$ is coupled with the approximation $u_{\epsilon_{N}}^{N}$ of the Navier-Stokes component, in a local way. The term in the Navier-Stokes equation takes the form (see system of equations $(P S-N S)$ in Section 2)

$$
\theta^{0, \epsilon_{N}} *\left(\left(u_{\epsilon_{N}}^{N}-v\right) S_{t}^{N}\right)
$$

and the corresponding term in the identity satisfied by the empirical measure $S_{t}^{N}$ (Lemma 3.2) has the form

$$
\left\langle S_{t}^{N},\left(u_{\epsilon_{N}}^{N}-v\right) \nabla_{v} \varphi\right\rangle .
$$

In order to pass to the limit in the previous terms we need uniform convergence of $u_{\epsilon_{N}}^{N}$ to $u$.

This is a demanding property that we approach, using Sobolev embedding theorem, by controlling the first derivatives of $u_{\epsilon_{N}}^{N}$. We approach it by means of the equation for the vorticity $\omega^{N}$. This strategy reveals a conceptual problem with physical content: the presence of particles in the fluid may produce vorticity. The estimates on the vorticity are far from being obvious, due to the interaction with the particles. The equation for the vorticity contains the interaction term

$$
\frac{1}{N} \sum_{i=1}^{N}\left(u_{\epsilon_{N}}^{N}\left(X_{t}^{i, N}\right)-V_{t}^{i, N}\right) \nabla^{\perp} \cdot \delta_{X_{t}^{i, N}}^{\epsilon_{N}}
$$

where $\delta_{X_{t}^{i, N}}^{\epsilon_{N}}$ is a smooth approximation of the delta Dirac $\delta_{X_{t}^{i, N}}$. Hence the term $\nabla^{\perp} \cdot \delta_{X_{t}^{i, N}}^{\epsilon_{N}}$ may induce a blow-up in the estimates, a priori. This is a key conceptual difficulty we had to overcome, among others of more technical nature. The fact that an infinitesimal particle in a fluid may produce vorticity is the topic of recent research, see [16]. These works are restricted to single particle for very difficult technical reasons; it may be that some link with the present research will be possible in the future after due progresses.

Thanks to the fact that $\nabla \omega^{N}$ has a control due to the viscous term, the energy type estimate leads to control the term

$$
\left\|\frac{1}{N} \sum_{i=1}^{N}\left(u_{\epsilon_{N}}^{N}\left(X_{t}^{i, N}\right)-V_{t}^{i, N}\right) \delta_{X_{t}^{i, N}}^{\epsilon_{N}}\right\|_{L^{2}\left(\mathbb{T}^{2}\right)} .
$$

This is not a simple task; just to mention, the trivial estimate

$$
\leq \frac{1}{N} \sum_{i=1}^{N}\left(u_{\epsilon_{N}}^{N}\left(X_{t}^{i, N}\right)-V_{t}^{i, N}\right)\left\|\delta_{X_{t}^{i, N}}^{\epsilon_{N}}\right\|_{L^{2}\left(\mathbb{T}^{2}\right)}
$$


leads to diverging quantities. This introduces a new ingredient with its own difficulties, as explained in the next subsection.

1.1.2. The Regularized Empirical Measure. We control the term (2) by introducing the regularized empirical measure $F_{t}^{N}(x, v)$

$$
F_{t}^{N}(x, v)=\theta^{\varepsilon_{N}} * S_{t}^{N}
$$

(see details in Sect. 2), inspired by works of Karl Oelschleger, see for instance [24]. It allows us to write

$$
\begin{aligned}
& \left|\frac{1}{N} \sum_{i=1}^{N}\left(u_{\epsilon_{N}}^{N}\left(t, X_{t}^{i, N}\right)-V_{t}^{i, N}\right) \delta_{X_{t}^{i, N}}^{\epsilon_{N}}(x)\right| \\
& \quad \leq\left\|u_{\epsilon_{N}}^{N}(t, \cdot)\right\|_{\infty} \frac{1}{N} \sum_{i=1}^{N} \delta_{X_{t}^{i, N}}^{\epsilon_{N}}(x)+\left|\frac{1}{N} \sum_{i=1}^{N} V_{t}^{i, N} \delta_{X_{t}^{i, N}}^{\epsilon_{N}}(x)\right| \\
& \quad=\left\|u_{\epsilon_{N}}^{N}(t, \cdot)\right\|_{\infty} \int_{\mathbb{R}^{2}} F_{t}^{N}(x, v) d v+\left|\int_{\mathbb{R}^{2}} v F_{t}^{N}(x, v) d v\right| .
\end{aligned}
$$

The proof of the last line is given in Lemma 5.3.

Now the problem is to prove suitable estimates on the regularized empirical measure $F_{t}^{N}(x, v)$. Controls on $S_{t}^{N}$ are essentially amounts to suitable estimates on the SDEs satisfied by particles, while a full treatment of $F_{t}^{N}(x, v)$ requires both SDEs properties and PDEs arguments applied to the identity satisfied by $F_{t}^{N}(x, v)$ (Lemma 3.2). This identity however is not closed; commutators appear and several technical difficulties arise, which perhaps are new here with respect to previous literature.

1.1.3. The Cut-Off and its Removal. We are able to perform the estimates outlined above only when a suitable cut-off on velocity is introduced; see $\chi_{R}(u)$ introduced in Sect. 3 and appearing in the rest of the paper. The idea is to use this truncated system as a bridge to the original one. By using the truncation in the interaction between particles and fluid we managed to produce an a priori bound independently on the number of particles $N$

$$
\left\|u^{N, R}\right\|_{\infty} \leq C_{R}, \quad(\text { Lemma 5.9) }
$$

which we used to obtain a suitable tightness criterion, needed for the convergence. We remark that this bound was only possible due to the presence of the cut-off, since the constant provided in (3) depends on the threshold $R$ of the truncation.

Therefore the preliminary result is that the PDE-particle system with cut-off converges to the PDE system with cut-off. However, by showing that the velocity field of the PDE system with the cut-off satisfies

$$
\left\|u^{R}\right\| \leq C \quad \text { (Proposition 5.13) }
$$

independently on $R$, it is possible to prove that the PDE system with cut-off is also solution without cutoff. In summary we can prove that the PDE-particle system with cut-off converges to the PDE system without cut-off, see Proposition 5.1. The proof of this step is organized differently from the previous description but here we have explained the concept behind the proof.

The final problem is to prove that the cut-off can be removed also from the approximating PDE-particle system. This seems to be a difficult question. Here we use a special trick.

To appreciate the difficulty and the trick, think for a second to a different problem where the approximations are not random. Assume we have proved that $u^{N, R}$ converges uniformly to the limit $u$. Since $u$ is uniformly bounded by a constant $R_{0}$ we deduce that, eventually in $N$, also $u^{N, R}$ is bounded, say, by $R_{0}+1$. Hence eventually in $N$, the function $u^{N, R}$ solves the equation without cut- off, hence it is equal to the unique solution $u^{N}$ of such equation. Next, consider the full approximating sequence $\left(u^{N}\right)_{N \geq 1}$ solving the equations without cut-off; this sequence converges uniformly to $u$, because the property of limit involves only the tail of the sequence and the tail coincides with the tail of the sequence $\left(u^{N, R}\right)_{N \geq 1}$, 
which we know to converge to $u$. This idea resemble us the method used to prove well-posedness of 3D Navier-Stokes equations with strong rotation, see for instance [15].

Unfortunately this simple idea does not work when the approximations are random. Forget about the fact that our convergence is in law; go to another probability space where it is almost sure. Thus, almost surely, eventually we may transfer the uniform bound $R_{0}$ of the limit solution to a bound $R_{0}+1$ for the approximations. But this time the "eventually" qualification is random! Hence, given a large $N$, we cannot claim that the stochastic process $u^{N, R}$ coincides with the unique solution $u^{N}$ of the equation without cut-off, because the bound on $u^{N, R}$ is true only for certain $\omega^{\prime}$ 's.

So the problem is that we have two families of stochastic processes, $\left(u^{N, R}\right)_{N>1}$ and $\left(u^{N}\right)_{N>1}$ and we know that for a.e. $\omega$ there is $N_{0}(\omega)$ such that for $N \geq N_{0}(\omega)$ the paths of the sequence $\left(u^{N, R}(\omega)\right)_{N \geq N_{0}(\omega)}$ are bounded, say, by $R_{0}+1<R$. We want to deduce a relation between $\left(u^{N, R}\right)_{N \geq 1}$ and $\left(u^{N}\right)_{N \geq 1}$ from this property. To this aim we invoke a property of path-by-path uniqueness (see [12]) opposite to the usual concept of pathwise uniqueness: given $\omega$, for $N \geq N_{0}(\omega)$ the path $u^{N, R}(\omega)$ satisfies the equation without cut-off (formulated for that single $\omega$ ) and by path-by-path uniqueness it coincides with $u^{N}(\omega)$. The conclusion is the same as in the deterministic case: consider the sequence of processes $\left(u^{N}\right)_{N \geq 1}$; for a.e. $\omega$, the sequence of functions $\left(u^{N}(\omega)\right)_{N \geq 1}$ converges to $u$ because it coincides, eventually, with the sequence $\left(u^{N, R}(\omega)\right)_{N \geq 1}$. The first major result of path-by-path uniqueness for SDEs has been proved by [13] and it is a very sophisticated result; however, here we have additive noise and relatively smooth coefficients, hence path-by-path uniqueness in our case is not difficult. We isolated the idea behind this reasoning into a general criterion, that we applied to transfer the convergence from the particle system where the cut-off is present, to the system without the cut-off.

The structure of this paper is the following: In Sect. 2 we introduce all the notation that we will use and we present our main result, Theorem 2.3. In Sect. 3 we collect some preliminary result that will be needed in the rest of the manuscript, while Sect. 4 is devoted to a theorem of uniqueness for the Vlasov-Navier-Stokes system. In Sect. 5 we prove a first intermediate result, that is the convergence of the particle system with the cut-off to the Vlasov-Navier-Stokes system without the cut off. Finally, in Sect. 6 we manage to remove the cut-off also from the approximating system, ending the proof of Theorem 2.3.

\section{Notation and Main Results}

We begin this section by introducing rigorously the Vlasov-Navier-Stokes system and its associated particle model. We will always assume the framework of a filtered probability space, denoted by $\left(\Omega, \mathcal{F},\left\{\mathcal{F}_{t}\right\}, \mathbf{P}\right)$. For the whole manuscript we will also work on the two dimensional torus $\mathbb{T}^{2}=\mathbb{R}^{2} / \mathbb{Z}^{2}$. The case of other bounded domains is more delicate due to creation of vorticity at the boundaries. Some of the intermediate results stated here will work also in higher dimension. However, to obtain the full result, due to uniqueness and smoothness obstacles, dimension $d=2$ is needed, so we will always keep the dimension fixed for a matter of simplicity.

We start by recalling the Vlasov-Navier-Stokes PDE-system

$$
\begin{cases}\partial_{t} u=\Delta u-u \cdot \nabla u-\nabla \pi-\int_{\mathbb{R}^{2}}(u-v) F(x, v) d v & (t, x) \in[0, T] \times \mathbb{T}^{2} \\ \partial_{t} F+v \cdot \nabla_{x} F+\operatorname{div}_{v}((u-v) F)=\frac{\sigma^{2}}{2} \Delta_{v} F & (t, x, v) \in[0, T] \times \mathbb{T}^{2} \times \mathbb{R}^{2} \\ \operatorname{div}(u)=0, & \end{cases}
$$

$\sigma>0$, with initial condition $u(0, \cdot)=u_{0}$ and $F(0, \cdot, \cdot)=F_{0}$. We also introduce the continuous-discrete Particle System approximating $(V N S)$ : 


$$
\left\{\begin{array}{l}
\partial_{t} u^{N}=\Delta u^{N}-u^{N} \cdot \nabla u^{N}-\nabla \pi^{N}-\frac{1}{N} \sum_{i=1}^{N}\left(u_{\varepsilon_{N}}^{N}\left(X_{t}^{i, N}\right)-V_{t}^{i, N}\right) \delta_{X_{t}^{i, N}}^{\varepsilon_{N}} \\
\operatorname{div}\left(u^{N}\right)=0 \\
\left\{\begin{array}{l}
d X_{t}^{i, N}=V_{t}^{i, N} d t \\
d V_{t}^{i, N}=\left(u_{\varepsilon_{N}}^{N}\left(X_{t}^{i, N}\right)-V_{t}^{i, N}\right) d t+\sigma d B_{t}^{i}
\end{array}\right.
\end{array}\right.
$$

with initial condition

$$
u^{N}(0, \cdot)=u_{0}, \quad\left(X_{0}^{i, N}, V_{0}^{i, N}\right) \stackrel{\mathcal{L} a w}{\sim} F(0, \cdot, \cdot) d x d v \text { i.i.d }
$$

namely the random variables $\left(X_{0}^{i, N}, V_{0}^{i, N}\right)$ are independent and identically distributed with density $F(0, x, v)$. In the previous equations, $\left(B_{t}^{i}\right)_{t \geq 0}$ is a sequence of independent Brownian motions, $\theta^{0}$ is a mollifier over the torus, $\varepsilon_{N} \in \mathbb{R}^{+}$is a sequence converging to zero, and

$$
\theta^{0, \varepsilon_{N}}(x):=\varepsilon_{N}^{-2} \theta^{0}\left(x / \varepsilon_{N}\right), \quad u_{\varepsilon_{N}}^{N}:=u * \theta^{0, \varepsilon_{N}}, \quad \delta_{X_{t}^{i, N}}^{\varepsilon_{N}}(x):=\theta^{0, \varepsilon_{N}}\left(x-X_{t}^{i, N}\right),
$$

All the hypothesis and requirements on the objects introduced above are collected in Sect. 2.3.

\subsection{Definition of Weak Solutions}

Definition 2.1 (Definition of weak solution of $(V N S)$ ). We say a pair $(u, F)$ is a weak solution of $(V N S)$ if the following conditions are satisfied:

a)

$$
\begin{aligned}
& u \in L^{\infty}\left([0, T] ; L^{2}\left(\mathbb{T}^{2}\right)\right) \cap L^{2}\left([0, T] ; H^{1}\left(\mathbb{T}^{2}\right)\right) ; \\
& F \in L^{\infty}\left([0, T] ; L^{1}\left(\mathbb{T}^{2} \times \mathbb{R}^{2}\right) \cap L^{\infty}\left(\mathbb{T}^{2} \times \mathbb{R}^{2}\right)\right), \quad F \geq 0 ; \\
& F|v|^{2} \in L^{\infty}\left([0, T] ; L^{1}\left(\mathbb{T}^{2} \times \mathbb{R}^{2}\right)\right) ;
\end{aligned}
$$

b) for all $\varphi \in C^{\infty}\left([0, T] \times \mathbb{T}^{2} ; \mathbb{R}^{2}\right)$ with $\operatorname{div} \varphi=0$ we have

$$
\begin{aligned}
& \left\langle u_{t}, \varphi_{t}\right\rangle=\left\langle u_{0}, \varphi_{0}\right\rangle+\int_{0}^{t}\left\langle u_{s}, \frac{\partial \varphi_{s}}{\partial s}\right\rangle d s+\int_{0}^{t}\left\langle u_{s}, \Delta \varphi_{s}\right\rangle d s+\int_{0}^{t}\left\langle u_{s} \cdot \nabla \varphi_{s}, u_{s}\right\rangle d s \\
& -\int_{0}^{t} \int_{\mathbb{R}^{2}} \int_{\mathbb{T}^{2}} \varphi_{s}(x)\left(u_{s}(x)-v\right) F_{s}(x, v) d x d v d s,
\end{aligned}
$$

c) for all $\psi \in C^{\infty}\left([0, T] \times \mathbb{T}^{2} \times \mathbb{R}^{2} ; \mathbb{R}\right)$ with compact support in $v$ we have

$$
\begin{gathered}
\left\langle F_{t}, \psi_{t}\right\rangle=\left\langle F_{0}, \psi_{0}\right\rangle+\int_{0}^{t}\left\langle F_{s}, \frac{\partial \psi_{s}}{\partial s}\right\rangle d s+\frac{\sigma^{2}}{2} \int_{0}^{t}\left\langle F_{s}, \Delta_{v} \psi_{s}\right\rangle d s \\
+\int_{0}^{t}\left\langle F_{s}, v \cdot \nabla_{x} \psi_{s}\right\rangle d s+\int_{0}^{t}\left\langle F_{s},\left(u_{s}-v\right) \cdot \nabla_{v} \psi_{s}\right\rangle d s ;
\end{gathered}
$$

Definition 2.2 (Definition of Bounded weak solution of $(V N S)$ ). We say a pair $(u, F)$ is a bounded weak solution of $(V N S)$ if it is a weak solution and

$$
u \in L^{\infty}\left([0, T] \times \mathbb{T}^{2}\right) .
$$

We refer to Theorem 4.1 for an uniqueness result for bounded weak solutions. Existence of bounded weak solutions for system $(V N S)$ will be obtained as a consequence of our main convergence result. 


\subsection{The Empirical Measure of the Particle System}

Before stating our main result we introduce some function spaces defined as follows. Given the space $E=\mathbb{T}^{2} \times \mathbb{R}^{2}$ we introduce

$$
\mathbf{P}_{1}(E)=\left\{\mu \text { probability measure on }(E, \mathcal{B}(E))\left|\int_{E}\right| x \mid \mu(d x)<\infty\right\}
$$

the space of all probability measure on the Borel sets of $E$, with finite first moment. We endow this space with the Wasserstein-1 metric, that can be defined equivalently as

$$
\mathcal{W}_{1}(\mu, \nu)=\sup _{[\varphi]_{L i p} \leq 1}\left|\int_{E} \varphi d \mu-\int_{E} \varphi d \nu\right|
$$

where $[\varphi]_{\text {Lip }}$ is the usual Lipschitz seminorm. Endowed with this metric the space $\mathbf{P}_{1}$ becomes a complete separable metric space, whose convergence implies the weak convergence of probability measures.

From now on, when $\mu$ is a measure and $f$ is a function, we will denote by $\langle f, \mu\rangle$ the integration in full space of $f$ with respect to $\mu$.

We now introduce the empirical measure of the particle system

$$
S_{t}^{N}=\sum_{i=1}^{N} \delta_{\left(X_{t}^{i, N}, V_{t}^{i, N}\right)}
$$

which is random measure on $(\Omega, \mathcal{F}, \mathbf{P})$, on the space $C\left([0, T] ; \mathbf{P}_{1}\left(\mathbb{T}^{2} \times \mathbb{R}^{2}\right)\right)$. We will consider a smoothed version of the empirical measure: let us introduce two functions $\theta^{0}: \mathbb{T}^{2} \rightarrow \mathbb{R}$ and $\theta^{1}: \mathbb{R}^{2} \rightarrow \mathbb{R}$ which are $C^{\infty}$, non negative and integrate one. Introduce also

$$
\theta(x, v):=\theta^{0}(x) \theta^{1}(v)
$$

which is a function on the product space $\mathbb{T}^{2} \times \mathbb{R}^{2}$. Consider then

$$
\theta^{\varepsilon_{N}}(x, v)=\varepsilon_{N}^{-2} \theta^{0}\left(\varepsilon_{N}^{-1} x\right) \varepsilon_{N}^{-2} \theta^{1}\left(\varepsilon_{N}^{-1} v\right)=\theta^{0, \varepsilon_{N}}(x) \theta^{1, \varepsilon_{N}}(v)
$$

and let us define

$$
F_{t}^{N}(x, v):=\theta^{\varepsilon_{N}} * S_{t}^{N}=\frac{1}{N} \sum_{i=1}^{N} \theta^{0, \varepsilon_{N}}\left(x-X_{t}^{i, N}\right) \theta^{1, \varepsilon_{N}}\left(v-V_{t}^{i, N}\right)
$$

the mollified empirical measure.

Remark 2.1. Note that the function $\theta^{0, \varepsilon_{N}}$ in the previous equation, appear in system $(P S-N S)$ in the coupling term.

In what follows and in the rest of the manuscript we will adopt the following notation for the moments on the $v$ component for the function $F$ :

$$
m_{k} F(x):=\int_{\mathbb{R}^{2}}|v|^{k} F(x, v) d v, \quad M_{k} F:=\int_{\mathbb{T}^{2}} \int_{\mathbb{R}^{2}}|v|^{k} F(x, v) d v d x .
$$

where $m_{k} F(x)$ is function over $\mathbb{T}^{2}$ while $M_{k} F \in \mathbb{R}$.

\subsection{Main Result}

We summarize all the main hypotheses of our framework:

1) $u_{0} \in H^{2}\left(\mathbb{T}^{2}\right)$;

2) $F_{0} \in\left(L^{1} \cap L^{\infty}\right)\left(\mathbb{T}^{2} \times \mathbb{R}^{2}\right)$ and $M_{6} F_{0}<\infty$;

3) $\theta(x, v)=\theta^{0}(x) \theta^{1}(v), \theta^{0}$ and $\theta^{1}$ mollifiers on $\mathbb{T}^{2}$ and $\mathbb{R}^{2}$ respectively, such that $\left|\nabla \theta^{0}(x)\right| \leq \theta^{0}(x)$ and $\operatorname{supp}\left(\theta^{1}\right) \subseteq B(0,1)$. Moreover $\theta^{1}(v)$ satisfies the following symmetry assumption $\int_{\mathbb{R}^{2}} \theta^{1}(v) v=0$;

4) The scaling factor $\varepsilon_{N}$ satisfies $\varepsilon_{N}=N^{-\beta}$ with $\beta \leq 1 / 4$; 
Remark 2.2. We remark that hypothesis (3) is needed in Lemma 5.5 to obtain the first a priori estimate on the mollified empirical measure. Regarding the scaling factor in (4), this hypothesis is also needed for Lemma 5.5: the bound on $\beta$ is strictly related to the space dimension and to the $L^{p}$ norm that is computed. In our case, we will compute the $L^{4}$ norm, and the general requirement in dimension $d$ is

$$
\beta \leq \frac{d}{3 d+2} .
$$

In what follows we will always use the notation $\lesssim$ to indicate that the inequality holds, up to a multiplicative constant that does not depend on any of the key parameters involved. To emphasize the dependence on one of those parameter we will adopt the convention $\lesssim_{X}$ to denote the dependence on the parameter $X$. Moreover we will make use of the letter $C$ to mark a constant, whose value does not matter for the argument.

We are finally able to present our main result:

Theorem 2.3. Under hypothesis of Sect. 2.3, the family of laws $\left\{Q^{N}\right\}_{N \in \mathbb{N}}$ of the couple $\left(u^{N}, S^{N}\right)_{N \in \mathbb{N}}$ is tight on $C\left([0, T] \times \mathbb{T}^{2}\right) \times C\left([0, T] ; \mathbf{P}_{1}\left(\mathbb{T}^{2} \times \mathbb{R}^{2}\right)\right)$. Moreover $\left\{Q^{N}\right\}_{N \in \mathbb{N}}$ converges weakly to $\delta_{(u, F)}$, where the couple $(u, F)$ is the unique bounded weak solution of system of equation (VNS).

\section{Preliminary Results}

In this section we collect the basic results about our particle systems, and all the technical inequality that will be used in the rest of the paper.

In order to obtain Theorem 2.3, it is necessary to introduce another coupled system of PDE-SDE, where the interaction between the particles and fluid is truncated. Introduce for $R>0$ the cut-off function $\chi_{R}^{0}: \mathbb{R} \rightarrow[0,1]$ defined as

$$
\chi_{R}^{0}(x)= \begin{cases}1 & \text { if } x \leq R-1 \\ 0 & \text { if } x \geq R\end{cases}
$$

and that is $C^{\infty}(\mathbb{R})$. Define also $\chi_{R}(u)=\chi_{R}^{0}\left(\|u\|_{L^{\infty}\left(\mathbb{T}^{2}\right)}\right)$. With this choice of notation one has

$$
\left\|u \chi_{R}(u)\right\|_{L^{\infty}\left(\mathbb{T}^{2}\right)} \leq R \text {. }
$$

Introduce now the truncated PDE-system:

$$
\left\{\begin{array}{l}
\partial_{t} u^{R}=\Delta u^{R}-u^{R} \cdot \nabla u^{R}-\nabla \pi-\int_{\mathbb{R}^{2}}\left(u^{R}-v\right) \chi_{R}\left(u_{t}^{R}\right) F^{R}(x, v) d v \\
\partial_{t} F^{R}=\frac{\sigma^{2}}{2} \Delta_{v} F^{R}-v \cdot \nabla_{x} F^{R}-\operatorname{div}_{v}\left(\left(u^{R} \chi_{R}\left(u^{R}\right)-v\right) F^{R}\right) \\
\operatorname{div}\left(u^{R}\right)=0
\end{array}\right.
$$

with the same initial conditions as system $(V N S)$. We also introduce the continuous-discrete truncated Particle System approximating $\left(V N S^{R}\right)$ :

$$
\begin{cases}\partial_{t} u^{N, R}=\Delta u^{N, R}-u^{N, R} \cdot \nabla u^{N, R}-\nabla \pi^{N, R} & \\
\quad-\frac{1}{N} \sum_{i=1}^{N}\left(u_{\varepsilon_{N}}^{N, R}\left(X_{t}^{i, N, R}\right)-V_{t}^{i, N, R}\right) \chi_{R}\left(u_{t}^{N, R}\right) \delta_{X_{t}^{i, N, R}}^{\varepsilon_{N}} & \\
\operatorname{div}\left(u^{N, R}\right)=0, & \\
\left\{\begin{array}{l}
d X_{t}^{i, N, R}=V_{t}^{i, N, R} d t \\
\left.d V_{t}^{i, N, R}=\left(u_{\varepsilon_{N}}^{N, R}\left(X_{t}^{i, N, R}\right) \chi_{R}\left(u_{t}^{N, R}\right)-V_{t}^{i, N ; R}\right) d t+\sigma d S_{t}^{R}\right)
\end{array}\right. & i=1, \ldots, N\end{cases}
$$

using the same notation and initial condition as $(P S-N S)$.

Definition 3.1 (Definition of bounded weak solution of $\left(V N S^{R}\right)$ ). We say a pair $\left(u^{R}, F^{R}\right)$ is a bounded weak solution of $\left(V N S^{R}\right)$ if the following condition are satisfied: 
a)

$$
\begin{aligned}
& u^{R} \in L^{\infty}\left([0, T] \times \mathbb{T}^{2}\right) \cap L^{2}\left([0, T] ; H^{1}\left(\mathbb{T}^{2}\right)\right) ; \\
& F^{R} \in L^{\infty}\left([0, T] ; L^{1}\left(\mathbb{T}^{2} \times \mathbb{R}^{2}\right) \cap L^{\infty}\left(\mathbb{T}^{2} \times \mathbb{R}^{2}\right)\right), \quad F^{R} \geq 0 ; \\
& F|v|^{2} \in L^{\infty}\left([0, T] ; L^{1}\left(\mathbb{T}^{2} \times \mathbb{R}^{2}\right)\right) ;
\end{aligned}
$$

b) for each divergence free, $C^{\infty}$ vector field $\varphi:[0, T] \times \mathbb{T}^{2} \rightarrow \mathbb{R}^{2}$ we have

$$
\begin{array}{r}
\left\langle u_{t}^{R}, \varphi_{t}\right\rangle=\left\langle u_{0}^{R}, \varphi_{0}\right\rangle+\int_{0}^{t}\left\langle u_{s}^{R}, \frac{\partial \varphi_{s}}{\partial s}\right\rangle d s+\int_{0}^{t}\left\langle u_{s}^{R}, \Delta \varphi_{s}\right\rangle d s+\int_{0}^{t}\left\langle u_{s}^{R} \cdot \nabla \varphi_{s}, u_{s}^{R}\right\rangle d s \\
+\int_{0}^{t}\left\langle\pi_{s}, \nabla \varphi_{s}\right\rangle d s-\int_{0}^{t} \int_{\mathbb{R}^{2}} \int_{\mathbb{T}^{2}} \varphi_{s}(x)\left(u_{s}^{R}(x)-v\right) \chi_{R}\left(u_{s}^{R}\right) F_{s}^{R}(x, v) d x d v d s
\end{array}
$$

c) for each $C^{\infty}$ function $\psi:[0, T] \times \mathbb{T}^{2} \times \mathbb{R}^{2} \rightarrow \mathbb{R}$, we have

$$
\begin{aligned}
& \left\langle F_{t}^{R}, \psi_{t}\right\rangle=\left\langle F_{0}^{R}, \psi_{0}\right\rangle+\int_{0}^{t}\left\langle F_{s}^{R}, \frac{\partial \psi_{s}}{\partial s}\right\rangle d s+\frac{\sigma^{2}}{2} \int_{0}^{t}\left\langle F_{s}^{R}, \Delta_{v} \psi_{s}\right\rangle d s \\
& \quad+\int_{0}^{t}\left\langle F_{s}^{R}, v \cdot \nabla_{x} \psi_{s}\right\rangle d s+\int_{0}^{t}\left\langle F_{s}^{R},\left(u_{s}^{R} \chi_{R}\left(u_{s}^{R}\right)-v\right) \cdot \nabla_{v} \psi_{s}\right\rangle d s
\end{aligned}
$$

Applying the maximum principle to system of equation $\left(V N S^{R}\right)$ we have

$$
\left\|F^{R}(t, x, v)\right\|_{L^{p}\left(\mathbb{T}^{2} \times \mathbb{R}^{2}\right)} \leq C_{T}\left\|F_{0}(x, v)\right\|_{L^{p}\left(\mathbb{T}^{2} \times \mathbb{R}^{2}\right)} \quad \forall p>1
$$

so that

$$
\left\|F^{R}(t, x, v)\right\|_{L^{\infty}\left(\mathbb{T}^{2} \times \mathbb{R}^{2}\right)} \leq C
$$

independently on $R$. We now introduce the empirical measure of the truncated particle system

$$
S_{t}^{N, R}=\sum_{i=1}^{N} \delta_{\left(X_{t}^{i, N, R}, V_{t}^{i, N, R}\right)}
$$

and its associated mollified empirical measure

$$
F_{t}^{N, R}(x, v)=\theta^{\varepsilon_{N}} * S_{t}^{N, R} .
$$

We now recall the identity satisfied by the empirical measure $S_{t}^{N}$.

Lemma 3.2. For every test function $\varphi: \mathbb{T}^{2} \times \mathbb{R}^{2} \rightarrow \mathbb{R}$ the empirical measure $S_{t}^{N}$ satisfies the following identity

$$
\begin{aligned}
d\left\langle S_{t}^{N}, \varphi\right\rangle= & \left\langle S_{t}^{N}, v \cdot \nabla_{x} \varphi\right\rangle d t+\left\langle S_{t}^{N},\left(u_{\varepsilon_{N}}^{N}-v\right) \cdot \nabla_{v} \varphi\right\rangle d t \\
& +\frac{\sigma^{2}}{2}\left\langle S_{t}^{N}, \Delta_{v} \varphi\right\rangle d t+d M_{t}^{N, \varphi},
\end{aligned}
$$

with

$$
M_{t}^{N, \varphi}=\frac{\sigma}{N} \sum_{i=1}^{N} \int_{0}^{t} \nabla_{v} \varphi\left(X_{t}^{i, N}, V_{t}^{i, N}\right) \cdot d B_{t}^{i} .
$$

Moreover $F_{t}^{N}(x, v)=\left(\theta^{\varepsilon_{N}} * S_{t}^{N}\right)(x, v)$ satisfies:

$$
\begin{aligned}
d F_{t}^{N}= & \frac{\sigma^{2}}{2} \Delta_{v} F_{t}^{N}-d i v_{v}\left(\theta^{\varepsilon_{N}} *\left(u_{\varepsilon_{N}}^{N}-v\right) S_{t}^{N}\right) d t \\
& -d i v_{x}\left(\theta^{\varepsilon_{N}} * v S_{t}^{N}\right) d t+d M_{t}^{N, \varepsilon_{N}},
\end{aligned}
$$

with $M_{t}^{N, \varepsilon_{N}}=M_{t}^{N, \theta^{\varepsilon_{N}}(x-\cdot, v-\cdot)}$.

Proof. The first part follows easily by applying Itô formula to $\varphi\left(X_{t}^{i, N}, V_{t}^{i, N}\right)$ and using linearity. The second part follows by taking $\varphi(\cdot, \cdot)=\theta^{\varepsilon_{N}}(x-\cdot, v-\cdot)$. 
The analogue of the previous result holds for the empirical measure of the truncated system $S^{N, R}$, as well as for it mollified version $F^{N, R}$. We now state the kinetic energy balance for the truncated system:

Lemma 3.3. With the previous notation, we denote with $\mathcal{E}^{N}$ the kinetic energy of the microscopic system,

$$
\mathcal{E}^{N}(t)=\frac{1}{2} \int_{\mathbb{T}^{2}}\left|u_{t}^{N, R}(x)\right|^{2} d x+\frac{1}{2 N} \sum_{i=1}^{N}\left|V_{t}^{i, N, R}\right|^{2} .
$$

One has formally

$$
\begin{aligned}
& \frac{1}{2} \frac{d}{d t} \mathcal{E}^{N}(t)+\int_{\mathbb{T}^{2}}\left|\nabla u_{t}^{N, R}(x)\right| d x d t \\
& \quad+\frac{1}{N} \sum_{i=1}^{N}\left|u_{\varepsilon_{N}}^{N, R}\left(X_{t}^{i, N, R}\right) \chi_{R}\left(u_{t}^{N, R}\right)-V_{t}^{i, N, R}\right|^{2} d t \leq \\
& \leq \frac{2 \sigma^{2}}{2} d t+\frac{\sigma}{N} \sum_{i=1}^{N} V_{t}^{i, N, R} \cdot d B_{t}^{i} .
\end{aligned}
$$

Proof. The lemma follows by Itô formula and by classical energy estimates for $u^{N, R}$.

Remark 3.1. The last inequality guarantees that, even if the truncated system has no direct interpretation for the dynamics of particle-fluid, it maintains the basics physical properties such as the conservation of the kinetic energy in the average.

An analogue of the previous result holds for the limit PDE system $\left(V N S^{R}\right)$, as well as for $(V N S)$. We state it in the case of system $\left(V N S^{R}\right)$ and omit the proof, which is classical.

Lemma 3.4. If $\left(u^{R}, F^{R}\right)$ is a weak solution of $\left(V N S^{R}\right)$, denoting with $\mathcal{E}$ the kinetic energy of the macroscopic system

$$
\mathcal{E}(t)=\frac{1}{2}\left(\int_{\mathbb{T}^{2}}\left|u_{t}^{R}\right|^{2} d x+\int_{\mathbb{R}^{2}} \int_{\mathbb{T}^{2}}|v|^{2} F_{t}^{R} d x d v\right)
$$

one has

$$
\frac{d}{d t} \mathcal{E}(t)+\int_{\mathbb{T}^{2}}\left|\nabla u_{t}^{R}\right|^{2} d x+\int_{\mathbb{R}^{2}} \int_{\mathbb{T}^{2}} F_{t}^{R}\left|u_{t}^{R}-v\right|^{2} \chi_{R}\left(u_{t}^{R}\right) d x d v=\frac{\sigma^{2}}{2}\left\|F_{0}\right\|_{L^{1}\left(\mathbb{R}^{2} \times \mathbb{T}^{2}\right)} .
$$

Moreover there exists a constant $C$, independent on $R$ such that

$$
\int_{0}^{T} \int_{\mathbb{R}^{2}} \int_{\mathbb{T}^{2}}|v|^{2} F_{t}^{R} d x d v d t \leq C .
$$

Remark 3.2. By the previous lemma we have a bound on $u^{R}$ in the norm $L^{2}\left([0, T] ; H^{1}\left(\mathbb{T}^{2}\right)\right)$ independently on $R$. By Sobolev embedding in dimension two we also have an uniform bound with respect to $R$ on $u^{R}$ in the space $L^{2}\left([0, T] ; L^{p}\left(\mathbb{T}^{2}\right)\right)$ for all $p<\infty$.

We now collect all the inequalities concerning the marginal distributions of the function $F$ : some of them are classical, see [20,27], while others have been used in [14].

Lemma 3.5. If $F$ is positive, defined on $\mathbb{T}^{2} \times \mathbb{R}^{2}$, the followings hold

1.

$$
\begin{aligned}
\left\|m_{0} F\right\|_{L^{2}\left(\mathbb{T}^{2}\right)}^{2} & \lesssim\left(\|F\|_{L^{\infty}\left(\mathbb{T}^{2} \times \mathbb{R}^{2}\right)}+1\right)^{2} M_{2} F, \\
\left\|m_{0} F\right\|_{L^{4}\left(\mathbb{T}^{2}\right)}^{4} & \lesssim\left(\|F\|_{L^{\infty}\left(\mathbb{T}^{2} \times \mathbb{R}^{2}\right)}+1\right)^{4} M_{6} F ;
\end{aligned}
$$

2.

$$
\left\|m_{1} F\right\|_{L^{2}\left(\mathbb{T}^{2}\right)}^{2} \lesssim\left(\|F\|_{L^{\infty}\left(\mathbb{T}^{2} \times \mathbb{R}^{2}\right)}+1\right)^{2} M_{4} F
$$


3.

$$
\left\|m_{0} F\right\|_{L^{2}\left(\mathbb{T}^{2}\right)}^{2} \lesssim\|F\|_{L^{4}\left(\mathbb{T}^{2} \times \mathbb{R}^{2}\right)}^{4}+M_{3} F
$$

4.

$$
\left\|m_{1} F\right\|_{L^{2}\left(\mathbb{T}^{2}\right)}^{2} \lesssim\|F\|_{L^{4}\left(\mathbb{T}^{2} \times \mathbb{R}^{2}\right)}^{4}+M_{6} F
$$

5. For all $k<k^{\prime}$

$$
M_{k} F \lesssim\|F\|_{L^{1}\left(\mathbb{T}^{2} \times \mathbb{R}^{2}\right)}+M_{k^{\prime}} F
$$

Proof. All the inequalities are derived through the same strategy: 1. and 2. are classical, see [20], while the proof of 3. can be found in [14], so we only outline the main idea. For inequality 1. and 3. let us consider the following decomposition

$$
\begin{aligned}
\int_{\mathbb{R}^{2}} F d v & =\int_{|v| \leq r(x)} F d v+\int_{|v|>r(x)} F d v \\
& \leq \int_{|v| \leq r(x)} F d v+\frac{1}{r(x)^{k}} \int_{|v|>r(x)}|v|^{k} F d v
\end{aligned}
$$

where $r(x)$ will be chosen in the next lines. Now one can estimates the integral on the ball of radius $r(x)$ using the infinity norm of $F$ for inequality 1 . or using Holder inequality to obtain $\|F\|_{L^{4}}$ for inequality 3 . To obtain the desired result, one has to take the square both sides, integrate on $\mathbb{T}^{2}$ and choose $r(x)$ in order to group all the terms. For inequality 2. and 4. one has just to decompose $\int|v| F d v$ and apply the same strategy, while for 5 is enough to take $r(x) \equiv 1$.

Remark 3.3. Inequality 3. and 4. will be used to prove a first tightness result in Sect. 5. Motivated by the fact that the infinity norm is not available on the mollified empirical measure, we propose a variant of 1 . and 2., avoiding the use of such norm. Inequalities 1 . and 2. will be used in Section 5 in order to prove a bound on the infinity norm of $u^{R}$, while 5 . will be used in the next lemma.

We now state and prove a variant of Lemma 2.1 in [20]. This variation is needed due to the presence of the noise on the diffusion on the particle velocity, i.e. the presence of $\Delta_{v}$ in the equation for $F^{R}$.

Lemma 3.6. If $\left(u^{R}, F^{R}\right)$ is a bounded weak solution of $\left(V N S^{R}\right), k>2$ and if $M_{k} F_{0}$ is finite, then there exists a constant $C_{k}$, independent on $R$, such that

$$
\sup _{t \in[0, T]} M_{k} F_{t}^{R} \leq C_{k} .
$$

The same result holds for any $(u, F)$ weak solutions of $(V N S)$.

Proof. In this proof we omit the superscript $R$ in $\left(u^{R}, F^{R}\right)$ to short the notation. We start by computing

$$
\begin{aligned}
\frac{d}{d t} \int_{\mathbb{R}^{2}} \int_{\mathbb{T}^{2}}|v|^{k} F_{t} d x d v \lesssim & \int_{\mathbb{T}^{2}}|u(t, x)| \int_{\mathbb{R}^{2}}|v|^{k-1} F_{t} d v d x+\int_{\mathbb{R}^{2}} \int_{\mathbb{T}^{2}}|v|^{k} F_{t} d x d v \\
& +\int_{\mathbb{R}^{2}} \int_{\mathbb{T}^{2}}|v|^{k-2} F_{t} d x d v .
\end{aligned}
$$

Following [20] we have

$$
\int_{\mathbb{T}^{2}}|u(t, x)| \int_{\mathbb{R}^{2}}|v|^{k-1} F_{t} d v d x \lesssim\left\|u_{t}\right\|_{L^{k+2}\left(\mathbb{T}^{2}\right)}\left(\int_{\mathbb{R}^{2}} \int_{\mathbb{T}^{2}}|v|^{k} F_{t} d x d v\right)^{1-\frac{1}{k+2}}
$$

while, using Lemma 3.5 inequality 5 . we have

$$
\int_{\mathbb{R}^{2}} \int_{\mathbb{T}^{2}}|v|^{k-2} F_{t} d x d v \leq \int_{\mathbb{R}^{2}} \int_{\mathbb{T}^{2}}|v|^{k} F_{t} d x d v+\left\|F_{t}\right\|_{L^{1}\left(\mathbb{T}^{2} \times \mathbb{R}^{2}\right)}
$$


Hence we get

$$
\begin{aligned}
M_{k} F_{t} \lesssim & M_{k} F_{0}+\int_{0}^{t}\left\|u_{s}\right\|_{L^{k+2}\left(\mathbb{T}^{2}\right)}\left(\int_{\mathbb{R}^{2}} \int_{\mathbb{T}^{2}}|v|^{k} F_{s} d x d v\right)^{1-\frac{1}{k+2}} d t \\
& +\int_{0}^{t} M_{k} F_{s} d s+C .
\end{aligned}
$$

We now note that

$$
\left(\int_{\mathbb{R}^{2}} \int_{\mathbb{T}^{2}}|v|^{k} F_{s} d x d v\right)^{1-\frac{1}{k+2}} \leq C\left(\int_{\mathbb{R}^{2}} \int_{\mathbb{T}^{2}}|v|^{k} F_{s} d x d v+1\right),
$$

hence we obtain

$$
\begin{aligned}
M_{k} F_{t} & \leq M_{k} F_{0}+C \int_{0}^{t}\left(\left\|u_{s}\right\|_{L^{k+2}\left(\mathbb{T}^{2}\right)}+1\right) M_{k} F_{s} d s+C \int_{0}^{t}\left(\left\|u_{s}\right\|_{L^{k+2}\left(\mathbb{T}^{2}\right)}+1\right) d s \leq \\
& \leq C\left(M_{k} F_{0}+\|u\|_{L^{2}\left([0, T] ; L^{k+2}\left(\mathbb{T}^{2}\right)\right)}\right)+C \int_{0}^{t}\left(\left\|u_{s}\right\|_{L^{k+2}\left(\mathbb{T}^{2}\right)}+1\right) M_{k} F_{s} d s .
\end{aligned}
$$

We conclude by classical Gronwall Lemma applied to the function $M_{k} F_{t}$ and by Remark 3.2.

\subsection{Maximum Principle for Weak Solutions of the Linear Vlasov-Fokker-Plank Equation}

We now focus on boundedness of weak solutions for the linear Vlasov-Fokker-Plank equation

$$
\partial_{t} F+v \cdot \nabla_{x} F+\operatorname{div}_{v}(a(t, x, v) F)=\Delta_{v} F .
$$

Boundedness of solutions will be fundamental in the latter when we will prove that the limit points, in the appropriate sense, of particle system $\left(P S^{R}-N S^{R}\right)$ are supported on bounded weak solutions of $(V N S)$.

While this topic is classical in the case of smooth solutions, the case of weak solutions is more delicate. What follows is mainly an adaptation of the work [8], Appendix A, Proposition A.3.

In that work the author assumed the vector field $a$ to be

$$
a \in L^{\infty}\left([0, T] \times \mathbb{T}^{2} \times \mathbb{R}^{2}\right), \quad \operatorname{div}_{v}(a) \in L^{\infty}\left([0, T] \times \mathbb{T}^{2} \times \mathbb{R}^{2}\right),
$$

and solutions $F$ are assumed to belong to the set

$$
Y:=\left\{F \in L^{2}\left([0, T] \times \mathbb{T}^{2} ; H^{1}\left(\mathbb{R}^{2}\right)\right) \text { s.t. } \partial_{t} F+v \cdot \nabla_{x} F \in L^{2}\left([0, T] \times \mathbb{T}^{2} ; H^{-1}\left(\mathbb{R}^{2}\right)\right)\right\} .
$$

On these solutions, a maximum principle is proved,

$$
\left\|F_{t}\right\|_{L^{\infty}\left(\mathbb{T}^{2} \times \mathbb{R}^{2}\right)} \leq C\left\|F_{0}\right\|_{L^{\infty}\left(\mathbb{T}^{2} \times \mathbb{R}^{2}\right)} .
$$

In our case, we have to consider

$$
a(t, x, v)=u(t, x)-v
$$

hence, we cannot apply directly the result presented in [8] since the function $a(t, x, v)$ is not globally bounded. However, it is possible to recover the same result by considering some estimates on higher moments for the function $F$. If $a$ satisfies (6), where $u$ is uniformly bounded, one can consider

$$
\begin{aligned}
& \tilde{Y}:=\left\{F \in L^{2}\left([0, T] \times \mathbb{T}^{2} ; H^{1}\left(\mathbb{R}^{2}\right)\right) \text { s.t. } v F \in L^{2}\left([0, T] \times \mathbb{T}^{2} \times \mathbb{R}^{2}\right),\right. \\
& \left.\partial_{t} F+v \cdot \nabla_{x} F \in L^{2}\left([0, T] \times \mathbb{T}^{2} ; H^{-1}\left(\mathbb{R}^{2}\right)\right)\right\} .
\end{aligned}
$$

Namely, in this setup the same result proved in [8] still works, provided that one can consider solutions satisfying

$$
\int_{0}^{T} \int_{\mathbb{T}^{2}} \int_{\mathbb{R}^{2}}|v|^{2} F_{s}^{2} d x d v d s<\infty .
$$


Without going into the details of this adaptation, we only remark that this additional condition is achievable under our hypothesis, since

$$
\begin{aligned}
& \int_{0}^{T} \int_{\mathbb{T}^{2}} \int_{\mathbb{R}^{2}}|v|^{2} F_{s}^{2} d x d v d s=\int_{0}^{T} \int_{\mathbb{T}^{2}} \int_{\mathbb{R}^{2}}|v|^{2} F_{s}^{\frac{1}{2}} F_{s}^{\frac{3}{2}} d x d v d s \\
& \leq\left(\int_{0}^{T} \int_{\mathbb{T}^{2}} \int_{\mathbb{R}^{2}}|v|^{4} F_{s} d x d v d s\right)^{\frac{1}{2}}\left(\int_{0}^{T} \int_{\mathbb{T}^{2}} \int_{\mathbb{R}^{2}} F_{s}^{3} d x d v d s\right)^{\frac{1}{2}},
\end{aligned}
$$

and we will show how to control the last two terms when needed.

\section{Uniqueness for Bounded Weak Solutiosn of System of Equations ( $V N S)$}

In this section we isolate a first major result needed to prove Theorem 2.3. We preferred to isolate it here, because it has some interest by itself. We present an uniqueness result for $(V N S)$ in the class of bounded weak solutions (Definition (2.2)). This result is required in order to prove that converging subsequences of the laws of $\left(u^{N_{k}}, S^{N_{k}}\right)$ are all supported on the same limit, which are in fact weak solutions of $(V N S)$.

Before going into the details of this Theorem let us make some remark about the hypothesis. We first highlight that the boundedness of solutions on the fluid component is strictly needed: we will make frequent of the fact that $u \in L^{\infty}\left([0, T] \times \mathbb{T}^{2}\right)$ in order to close some of the estimates needed to end the proof. We also remark that, even if in the proof we used the uniform bound $\|u\|_{L^{\infty}\left([0, T] \times \mathbb{T}^{2}\right)}$, with a bit more effort it is possible to complete the proof using only $u \in L^{2}\left([0, T] ; L^{\infty}\left(\mathbb{T}^{2}\right)\right)$. Motivated by the fact that in this work we prove existence of solutions uniformly bounded in time and space, we prefer to choose $u \in L^{\infty}\left([0, T] \times \mathbb{T}^{2}\right)$. Regarding the assumptions on weak derivatives, we require only

$$
u \in L^{\infty}\left([0, T] \times \mathbb{T}^{2}\right) \cap L^{2}\left([0, T] ; H^{1}\left(\mathbb{T}^{2}\right)\right)
$$

avoiding any assumption on the second derivative of $u$.

Also in the following proof we will make frequent use of Gagliardo-Nirenberg inequality in dimension two

$$
\|u\|_{L^{p}} \lesssim\|u\|_{L^{2}}^{\frac{2}{p}}\|\nabla u\|_{L^{2}}^{\frac{2}{q}}
$$

where $\frac{1}{p}+\frac{1}{q}=\frac{1}{2}$. However, this is only needed to minimize the hypothesis on $M_{k} F_{0}$, required to be finite only for some $k$ strictly bigger than 4 . One could have used the classical Ladyzhenskaya's inequality $(p=q=4)$ with the downside of requiring higher-order moments to be finite.

The proof of this result is mainly inspired by the work [6].

Theorem 4.1. Let $\left(u_{1}, F_{1}\right)$ and $\left(u_{2}, F_{2}\right)$ be two bounded weak solutions (Definition 2.2) with the same initial conditions, of system $(V N S)$. If

$$
M_{4+\varepsilon} F_{i}(0)<\infty
$$

for some $\varepsilon>0$, then $u_{1}=u_{2}$ and $F_{1}=F_{2}$.

Proof. We introduce the new variables $F=F_{1}-F_{2}$ and $u=u_{1}-u_{2}$. Then the pair $(u, F)$ satisfies in the weak sense

$$
\begin{aligned}
& \partial_{t} u=\Delta u-u \cdot \nabla u_{1}-u_{2} \cdot \nabla u-\nabla\left(\pi_{1}-\pi_{2}\right)-\int_{\mathbb{R}^{2}}\left(u F_{1}+u_{2} F-v F\right) d v, \\
& \partial_{t} F=\Delta_{v} F-v \cdot \nabla_{x} F-\operatorname{div}_{v}\left(u F_{1}+u_{2} F-v F\right)
\end{aligned}
$$

with $(u(0, \cdot), F(0, \cdot, \cdot))=0$. We prove uniqueness by applying Gronwall Lemma to the quantity

$$
\left\|u_{t}\right\|_{L^{2}\left(\mathbb{T}^{2}\right)}^{2}+\left\|\langle v\rangle^{k} F_{t}\right\|_{L^{2}\left(\mathbb{T}^{2} \times \mathbb{R}^{2}\right)}^{2}
$$

for some $k>2$ which will be chosen later and where $\langle v\rangle=\left(1+|v|^{2}\right)^{\frac{1}{2}}$. 
We start by estimating $\left\|u_{t}\right\|_{L^{2}\left(\mathbb{T}^{2}\right)}^{2}$ : computing the time derivative we have

$$
\begin{aligned}
& \frac{d}{d t}\|u\|_{L^{2}\left(\mathbb{T}^{2}\right)}^{2}+\|\nabla u\|_{L^{2}\left(\mathbb{T}^{2}\right)}^{2} \\
& \quad \lesssim-\int_{\mathbb{T}^{2}} u\left(u \cdot \nabla u_{1}\right) d x-\int_{\mathbb{T}^{2}} u\left(u_{2} \cdot \nabla u\right) d x \\
& \quad-\int_{\mathbb{T}^{2}} u \int_{\mathbb{R}^{2}} v F d v d x-\int_{\mathbb{T}^{2}} u \int_{\mathbb{R}^{2}} u F_{1} d v d x-\int_{\mathbb{T}^{2}} u \int_{\mathbb{R}^{2}} u_{2} F d v d x
\end{aligned}
$$

Integrating by parts the term

$$
\int_{\mathbb{T}^{2}} u\left(u_{2} \cdot \nabla u\right) d x=0
$$

vanishes, while the term

$$
-\int_{\mathbb{T}^{2}} u \int_{\mathbb{R}^{2}} u F_{1} d v d x=-\int_{\mathbb{T}^{2}} \int_{\mathbb{R}^{2}} u^{2} F_{1} d v d x \leq 0
$$

can be neglected due to positivity of $F_{1}$. Hence we can estimate the remaining terms as

$$
\begin{aligned}
(7) \lesssim & -\int_{\mathbb{T}^{2}} u\left(u \cdot \nabla u_{1}\right) d x-\int_{\mathbb{T}^{2}} u \int_{\mathbb{R}^{2}} v F d v d x \\
& -\int_{\mathbb{T}^{2}} u \int_{\mathbb{R}^{2}} u_{2} F d v d x=(I)+(I I)+(I I I) .
\end{aligned}
$$

where

$$
\begin{aligned}
(I) & \leq \int_{\mathbb{T}^{2}}|u||\nabla u|\left|u_{1}\right| d x \leq\left\|u_{1}\right\|_{\infty} \int_{\mathbb{T}^{2}}|u||\nabla u| d x \\
& \lesssim \frac{1}{\delta}\|u\|_{L^{2}\left(\mathbb{T}^{2}\right)}^{2}+\delta\|\nabla u\|_{L^{2}\left(\mathbb{T}^{2}\right)}^{2}
\end{aligned}
$$

and $\delta>0$ can be taken arbitrarily small.

$$
\begin{aligned}
(I I) & \leq \int_{\mathbb{R}^{2}} \int_{\mathbb{T}^{2}}|u||v| F d x d v \leq \int_{\mathbb{R}^{2}} \int_{\mathbb{T}^{2}} \frac{|u|}{\langle v\rangle^{k-1}}\langle v\rangle^{k} F d x d v \\
& \leq \int_{\mathbb{T}^{2}}|u|^{2} d x \int_{\mathbb{R}^{2}} \frac{1}{\langle v\rangle^{2(k-1)}} d v+\left\|\langle v\rangle^{k} F\right\|_{L^{2}\left(\mathbb{T}^{2} \times \mathbb{R}^{2}\right)}^{2} \\
& \lesssim\|u\|_{L^{2}\left(\mathbb{T}^{2}\right)}^{2}+\left\|\langle v\rangle^{k} F\right\|_{L^{2}\left(\mathbb{T}^{2} \times \mathbb{R}^{2}\right)}^{2}
\end{aligned}
$$

because $2(k-1)>2$ being $k>2$.

$$
\begin{aligned}
(I I I) & \leq \int_{\mathbb{R}^{2}} \int_{\mathbb{T}^{2}}|u|\left|u_{2}\right| F d x d v \leq\left\|u_{2}\right\|_{\infty} \int_{\mathbb{R}^{2}} \int_{\mathbb{T}^{2}} \frac{|u|}{\langle v\rangle^{k}}\langle v\rangle^{k} F d x d v \\
& \lesssim \int_{\mathbb{T}^{2}}|u|^{2} d x \int_{\mathbb{R}^{2}} \frac{1}{\langle v\rangle^{2 k}} d v+\left\|\langle v\rangle^{k} F\right\|_{L^{2}\left(\mathbb{T}^{2} \times \mathbb{R}^{2}\right)}^{2} \lesssim\|u\|_{L^{2}\left(\mathbb{T}^{2}\right)}^{2}+\left\|\langle v\rangle^{k} F\right\|_{L^{2}\left(\mathbb{T}^{2} \times \mathbb{R}^{2}\right)}^{2}
\end{aligned}
$$


This ends the estimate for $\|u\|_{L^{2}\left(\mathbb{T}^{2}\right)}^{2}$. Concerning $\left\|\langle v\rangle^{k} F\right\|_{L^{2}\left(\mathbb{T}^{2} \times \mathbb{R}^{2}\right)}^{2}$ we proceed by computing the time derivative

$$
\begin{aligned}
\frac{d}{d t} & \left\|\langle v\rangle^{k} F\right\|_{L^{2}\left(\mathbb{T}^{2} \times \mathbb{R}^{2}\right)}^{2}+\left\|\langle v\rangle^{k} \nabla_{v} F\right\|_{L^{2}\left(\mathbb{T}^{2}\right)}^{2} \\
\lesssim & +\int_{\mathbb{R}^{2}} \int_{\mathbb{T}^{2}}\langle v\rangle^{2 k-2} F^{2} d x d v-\int_{\mathbb{R}^{2}} \int_{\mathbb{T}^{2}}\langle v\rangle^{2 k} F v \cdot \nabla_{x} F d x d v \\
& -\int_{\mathbb{R}^{2}} \int_{\mathbb{T}^{2}}\langle v\rangle^{2 k} F \operatorname{div}_{v}\left(u_{2} F\right) d x d v-\int_{\mathbb{R}^{2}} \int_{\mathbb{T}^{2}}\langle v\rangle^{2 k} F \operatorname{div}_{v}\left(u F_{1}\right) d x d v \\
& +\int_{\mathbb{R}^{2}} \int_{\mathbb{T}^{2}}\langle v\rangle^{2 k} F \operatorname{div}_{v}(v F) d x d v .
\end{aligned}
$$

The first term on the r.h.s. can be estimated with $\left\|\langle v\rangle^{k} F\right\|_{L^{2}\left(\mathbb{T}^{2} \times \mathbb{R}^{2}\right)}^{2}$, being $\langle v\rangle \geq 1$. By a standard integration by parts argument, it is proved that the second term is equal to zero. Hence, what is left from (8) is

$$
\begin{aligned}
& -\int_{\mathbb{R}^{2}} \int_{\mathbb{T}^{2}}\langle v\rangle^{2 k} F \operatorname{div}_{v}\left(u_{2} F\right) d x d v-\int_{\mathbb{R}^{2}} \int_{\mathbb{T}^{2}}\langle v\rangle^{2 k} F \operatorname{div}_{v}\left(u F_{1}\right) d x d v \\
& \quad+\int_{\mathbb{R}^{2}} \int_{\mathbb{T}^{2}}\langle v\rangle^{2 k} F \operatorname{div}_{v}(v F) d x d v=(I V)+(V)+(V I) .
\end{aligned}
$$

Now we proceed by treating each term separately:

$$
\begin{aligned}
(I V)= & -\frac{1}{2} \int_{\mathbb{R}^{2}} \int_{\mathbb{T}^{2}}\langle v\rangle^{2 k} u_{2} \cdot \nabla_{v} F^{2} d x d v \leq \int_{\mathbb{R}^{2}} \int_{\mathbb{T}^{2}}\langle v\rangle^{2 k-1}\left|u_{2}\right| F^{2} d x d v \\
\lesssim & \left\|u_{2}\right\|_{\infty} \int_{\mathbb{R}^{2}} \int_{\mathbb{T}^{2}}\langle v\rangle^{2 k} F^{2} d x d v \lesssim\left\|\langle v\rangle^{k} F\right\|_{L^{2}\left(\mathbb{T}^{2} \times \mathbb{R}^{2}\right)}^{2} \cdot \\
(V)= & \int_{\mathbb{R}^{2}} \int_{\mathbb{T}^{2}} \nabla_{v}\left(\langle v\rangle^{2 k} F\right) \cdot u F_{1} d x d v \leq \int_{\mathbb{R}^{2}} \int_{\mathbb{T}^{2}}\langle v\rangle^{2 k-1} F|u| F_{1} d x d v \\
& +\int_{\mathbb{R}^{2}} \int_{\mathbb{T}^{2}}\langle v\rangle^{2 k}\left|\nabla_{v} F\right||u| F_{1} d x d v .
\end{aligned}
$$

The first term on the r.h.s. of the last inequality can be treated in the following way

$$
\begin{gathered}
\int_{\mathbb{R}^{2}} \int_{\mathbb{T}^{2}}\langle v\rangle^{2 k-1} F|u| F_{1} d x d v=\int_{\mathbb{R}^{2}} \int_{\mathbb{T}^{2}}\left(\langle v\rangle^{k} F\right) \frac{|u|}{\langle v\rangle}\left(\langle v\rangle^{k} F_{2}\right) d x d v \\
\leq\left\|\langle v\rangle^{k} F\right\|_{L^{2}\left(\mathbb{T}^{2} \times \mathbb{R}^{2}\right)}\|u\|_{L^{p}\left(\mathbb{T}^{2}\right)}\left(\int_{\mathbb{R}^{2}} \frac{1}{\langle v\rangle^{p}} d v\right)^{\frac{1}{p}}\left\|\langle v\rangle^{k} F_{2}\right\|_{L^{q}\left(\mathbb{T}^{2} \times \mathbb{R}^{2}\right)}
\end{gathered}
$$

where $p$ and $q$ are such that $\frac{1}{p}+\frac{1}{q}+\frac{1}{2}=1$. Note that $p>2$ so that $1 /\langle v\rangle^{p}$ is integrable in dimension two. Applying Gagliardo-Niremberg inquality to the previous identity we have

$$
\begin{aligned}
(9) & \leq\left\|\langle v\rangle^{k} F\right\|_{L^{2}\left(\mathbb{T}^{2} \times \mathbb{R}^{2}\right)}\|u\|_{L^{2}\left(\mathbb{T}^{2}\right)}^{\frac{2}{p}}\|\nabla u\|_{L^{2}\left(\mathbb{T}^{2}\right)}^{\frac{2}{q}}\left\|\langle v\rangle^{k} F_{2}\right\|_{L^{q}\left(\mathbb{T}^{2} \times \mathbb{R}^{2}\right)} \\
& \lesssim\left\|\langle v\rangle^{k} F\right\|_{L^{2}\left(\mathbb{T}^{2} \times \mathbb{R}^{2}\right)}^{2}+\frac{1}{\delta}\|u\|_{L^{2}\left(\mathbb{T}^{2}\right)}^{2}+\delta\left\|\langle v\rangle^{k} F_{2}\right\|_{L^{q}\left(\mathbb{T}^{2} \times \mathbb{R}^{2}\right)}^{q}\left\|\nabla_{u}\right\|_{L^{2}\left(\mathbb{T}^{2}\right)}^{2}
\end{aligned}
$$

where $\delta$ can be taken arbitrarily small. In order to control the quantity $\left\|\langle v\rangle^{k} F_{2}\right\|_{L^{q}\left(\mathbb{T}^{2} \times \mathbb{R}^{2}\right)}^{q}$ at the end of the proof we will impose that $k q<4+\varepsilon$. On the other hand for the second term on the r.h.s. of $(V)$, 
introduce $\alpha>0$ such that $\alpha p>2$ so that

$$
\begin{aligned}
& \int_{\mathbb{R}^{2}} \int_{\mathbb{T}^{2}}\langle v\rangle^{2 k}\left|\nabla_{v} F\right||u| F_{1} d x d v=\int_{\mathbb{R}^{2}} \int_{\mathbb{T}^{2}}\langle k\rangle\left|\nabla_{v} F\right| \frac{|u|}{\langle v\rangle^{\alpha}}\langle v\rangle^{k+\alpha} F_{1} d x d v \\
& \leq\left\|\left.\langle v\rangle^{k}\left|\nabla_{v} F\right|\right|_{L^{2}\left(\mathbb{T}^{2} \times \mathbb{R}^{2}\right)}\right\| u\left\|_{L^{p}\left(\mathbb{T}^{2}\right)}\left(\int_{\mathbb{R}^{2}} \frac{1}{\langle v\rangle^{\alpha p}} d v\right)^{\frac{1}{p}}\right\|\langle v\rangle^{k+\alpha} F_{1} \|_{L^{q}\left(\mathbb{T}^{2} \times \mathbb{R}^{2}\right)} .
\end{aligned}
$$

Now we apply Gagliardo-Niremberg inequality and Young inequality, in the same manner as we treated (9), obtaining

$$
\lesssim \delta\left\|\langle v\rangle^{k}\left|\nabla_{v} F\right|\right\|_{L^{2}\left(\mathbb{T}^{2} \times \mathbb{R}^{2}\right)}^{2}+\frac{1}{\delta^{2}}\|u\|_{L^{2}\left(\mathbb{T}^{2}\right)}^{2}+\delta\left\|\langle v\rangle^{k+\alpha} F_{1}\right\|_{L^{q}\left(\mathbb{T}^{2} \times \mathbb{R}^{2}\right)}^{q}\|\nabla u\|_{L^{2}\left(\mathbb{T}^{2}\right)}^{2} .
$$

We require that $(k+\alpha) q<4+\varepsilon$ in order to match our hypothesis on $M_{4+\varepsilon} F(0)$. This ends the term in $(V)$. For the last one, by the product rule

$$
(V I) \lesssim\left\|\langle v\rangle^{k} F\right\|_{L^{2}\left(\mathbb{R}^{2} \times \mathbb{T}^{2}\right)}^{2}+\int_{\mathbb{R}^{2}} \int_{\mathbb{T}^{2}}\langle v\rangle^{2 k+1} \nabla_{v}\left(F^{2}\right) d x d v \lesssim\left\|\langle v\rangle^{k} F\right\|_{L^{2}\left(\mathbb{R}^{2} \times \mathbb{T}^{2}\right)}^{2} .
$$

What is left, before applying Gronwall Lemma, is only to find parameters $(k, p, q, \alpha)$ matching all the needed constraints:

$$
\left\{\begin{array}{l}
k>2 \\
\frac{1}{p}+\frac{1}{q}+\frac{1}{2}=1 ; \\
\alpha p>2 \\
(k+\alpha) q<4+\varepsilon .
\end{array}\right.
$$

The rationale behind this is the following: $k$ and $q$ can be taken arbitrarily close to 2 . Doing so, $p$ will be very large and hence $\alpha$ can be take arbitrarily small preserving the condition $\alpha p>2$, and having $(k+\alpha)$ close to 2 .

These conditions allow us obtain that

$$
\left\|\langle v\rangle^{k+\alpha} F_{1}\right\|_{L^{q}\left(\mathbb{T}^{2} \times \mathbb{R}^{2}\right)}^{q},\left\|\langle v\rangle^{k} F_{2}\right\|_{L^{q}\left(\mathbb{T}^{2} \times \mathbb{R}^{2}\right)}^{q}, \int_{\mathbb{R}^{2}} \frac{1}{\langle v\rangle^{\alpha p}} d v \leq C,
$$

being

$$
\begin{aligned}
\left\|\langle v\rangle^{k+\alpha} F_{1}\right\|_{L^{q}\left(\mathbb{T}^{2} \times \mathbb{R}^{2}\right)}^{q} & \leq\left\|F_{1}\right\|_{L^{\infty}\left(\mathbb{T}^{2} \times \mathbb{R}^{2}\right)}^{q-1} \int_{\mathbb{R}^{2}} \int_{\mathbb{T}^{2}}\langle v\rangle^{(k+\alpha) q} F_{1} d x d v \\
& \lesssim\left\|F_{1}\right\|_{L^{\infty}\left(\mathbb{T}^{2} \times \mathbb{R}^{2}\right)}^{q-1}\left(\left\|F_{1}\right\|_{L^{1}\left(\mathbb{T}^{2} \times \mathbb{R}^{2}\right)}+M_{(k+\alpha) q} F_{1}\right) \leq C .
\end{aligned}
$$

Summarizing we have obtained

$$
\begin{aligned}
& \frac{d}{d t}\left\|u_{t}\right\|_{L^{2}\left(\mathbb{T}^{2}\right)}^{2}+\frac{d}{d t}\left\|\langle v\rangle^{k} F_{t}\right\|_{L^{2}\left(\mathbb{T}^{2} \times \mathbb{R}^{2}\right)}^{2} \leq \\
& \quad \leq C_{1}\left\|u_{t}\right\|_{L^{2}\left(\mathbb{T}^{2}\right)}^{2}+C_{2}\left\|\langle v\rangle^{k} F_{t}\right\|_{L^{2}\left(\mathbb{T}^{2} \times \mathbb{R}^{2}\right)}^{2},
\end{aligned}
$$

hence by Gronwall Lemma we obtain $u \equiv 0$ and $F \equiv 0$, proving uniqueness.

\section{Scaling Limit for the Truncated System}

In this section we focus on the proof of a first tightness result. As remarked in the introduction, first we will prove the convergence of $\left(P S^{R}-N S^{R}\right)$ to $(V N S)$. To do so, we will show that, if the cutoff threshold $R$ is large enough, then the system $\left(V N S^{R}\right)$ coincides with $(V N S)$. This whole section is devoted to the proof of this intermediate result: 
Proposition 5.1. Under hypothesis of Sect. 2.3 and if $R \geq \mathbf{K}_{u}+1$, where the constant $\mathbf{K}_{u}$ will be specified later (Proposition 5.13), the family of laws $\left\{Q^{N, R}\right\}$ of the couple $\left(u^{N, R}, S^{N, R}\right)_{N \in \mathbb{N}}$ is tight on $C([0, T] \times$ $\left.\mathbb{T}^{2}\right) \times C\left([0, T] ; \mathbf{P}_{1}\left(\mathbb{T}^{2} \times \mathbb{R}^{2}\right)\right)$. Moreover $\left\{Q^{N, R}\right\}_{N \in \mathbb{N}}$ converges weakly to $\delta_{(u, F)}$, where the couple $(u, F)$ is the unique weak solution of system of equation (VNS).

With a special argument we will be finally able to remove the cut-off also in the approximating system and to get our main result, Theorem 2.3.

\subsection{Tightness}

In order to prove Proposition 5.1 we have to establish the tightness of the laws of the empirical measure $S^{N, R}$ and that of $u^{N, R}$. First we deal with the empirical measure, the easier of the two. The tightness of $S^{N, R}$ follows easily by a well known criterion, [26], being the particles exchangeable and due to the presence of the cut-off.

Proposition 5.2. The family of laws $\left\{Q^{N, R, S}\right\}_{N \in \mathbb{N}}$ of the empirical measure $\left\{S^{N, R}\right\}_{N \in \mathbb{N}}$ is relatively compact with respect of the weak convergence on $C\left([0, T] ; \mathbf{P}_{1}\left(\mathbb{T}^{2} \times \mathbb{R}^{2}\right)\right)$.

We now focus on the tightness of the laws of $u^{N, R}$. To get an idea of what is the right topology to work with, we focus on the coupling term that appears in the equation for $u^{N, R}$ in $\left(P S^{R}-N S^{R}\right)$. The term can be rewritten as

$$
\begin{aligned}
& \chi_{R}\left(u_{t}^{N, R}\right) \frac{1}{N} \sum_{i=1}^{N}\left(u_{\varepsilon_{N}}^{N, R}\left(X_{t}^{i, N, R}\right)-V_{t}^{i, N, R}\right) \delta_{X_{t}^{i, N, R}}^{\varepsilon_{N}} \\
& \quad=\chi_{R}\left(u_{t}^{N, R}\right) \int_{\mathbb{R}^{2}} \int_{\mathbb{T}^{2}}\left(u_{\varepsilon_{N}}^{N, R}\left(x^{\prime}\right)-v^{\prime}\right) \theta^{0, \varepsilon_{N}}\left(x-x^{\prime}\right) S_{t}^{N, R}\left(d x^{\prime}, d v^{\prime}\right)= \\
& \quad=\chi_{R}\left(u_{t}^{N, R}\right)\left(\theta^{0, \varepsilon_{N}} *\left(u_{\varepsilon_{N}}^{N, R}-v\right) S_{t}^{N, R}\right)(x) .
\end{aligned}
$$

In order to pass to the limit in the previous term, it is required that $u^{N, R}$ is converging uniformly over $\mathbb{T}^{2}$, since $S^{N, R}$ is converging only weakly as probability measure. Hence, we look for a tightness criterion for $\left\{u^{N, R}\right\}_{N \in \mathbb{N}}$ in $C\left(\mathbb{T}^{2}\right)$. By Sobolev embedding in dimension two we have $H^{2}\left(\mathbb{T}^{2}\right) \hookrightarrow C\left(\mathbb{T}^{2}\right.$ ) (and also in the space of holder continuous functions). Thus, to get estimates on second derivative of $u^{N, R}$, we start by looking at the equation for $u^{N, R}$ in vorticity form:

$$
\begin{aligned}
& \partial_{t} \omega^{N, R}=\Delta \omega^{N, R}-u^{N, R} \cdot \nabla \omega^{N, R} \\
& \left.\quad-\frac{\chi_{R}\left(u_{t}^{N, R}\right)}{N} \sum_{i=1}^{N}\left(u_{\varepsilon_{N}}^{N, R}\left(X_{t}^{i, N, R}\right)-V_{t}^{i, N, R}\right)\right) \nabla^{\perp} \cdot \delta_{X_{t}^{i, N, R}}^{\varepsilon_{N}} .
\end{aligned}
$$

In order to be able to obtain a priori estimates on $\omega^{N, R}$ we need first to rewrite the coupling term in (10) as a function of the mollified empirical measure $F^{N, R}$. We highlight that this is one of the most important key steps in this work, that remarks the importance to introduce the mollified empirical measure, and justify all the following computations.

\section{Lemma 5.3 .}

$$
\begin{aligned}
& \frac{1}{N} \sum_{i=1}^{N} V_{t}^{i, N, R} \delta_{X_{t}^{i, N, R}}^{\varepsilon_{N}}(x)=\int_{\mathbb{R}^{2}} v F_{t}^{N, R}(x, v) d v=m_{1} F_{t}^{N, R}(x) \\
& \frac{1}{N} \sum_{i=1}^{N} \delta_{X_{t}^{i, N, R}}^{\varepsilon_{N}}(x)=\int_{\mathbb{R}^{2}} F_{t}^{N, R}(x, v) d v=m_{0} F_{t}^{N, R}(x)
\end{aligned}
$$


Proof. Notice that

$$
\begin{gathered}
\frac{1}{N} \sum_{i=1}^{N} V_{t}^{i, N, R} \delta_{X_{t}^{i, N, R}}^{\varepsilon_{N}}(x)=\int_{\mathbb{R}^{2}} \int_{\mathbb{T}^{2}} \theta^{0, \varepsilon_{N}}\left(x-x^{\prime}\right) v^{\prime} S_{t}^{N, R}\left(d x^{\prime}, d v^{\prime}\right) \\
\quad=\int_{\mathbb{R}^{2}} \int_{\mathbb{R}^{2}} \int_{\mathbb{T}^{2}} \theta^{0, \varepsilon_{N}}\left(x-x^{\prime}\right) \theta^{1, \varepsilon_{N}}\left(v-v^{\prime}\right) v^{\prime} S_{t}^{N, R}\left(d x^{\prime}, d v^{\prime}\right) d v
\end{gathered}
$$

and

$$
\int_{\mathbb{R}^{2}} v F_{t}^{N, R}(x, v) d v=\int_{\mathbb{R}^{2}} \int_{\mathbb{R}^{2}} \int_{\mathbb{T}^{2}} \theta^{0, \varepsilon_{N}}\left(x-x^{\prime}\right) \theta^{1, \varepsilon_{N}}\left(v-v^{\prime}\right) v S_{t}^{N, R}\left(d x^{\prime}, d v^{\prime}\right) d v
$$

so that to complete the proof we only need to prove

$$
\int_{\mathbb{R}^{2}} \int_{\mathbb{R}^{2}} \int_{\mathbb{T}^{2}} \theta^{0, \varepsilon_{N}}\left(x-x^{\prime}\right) \theta^{1, \varepsilon_{N}}\left(v-v^{\prime}\right)\left(v-v^{\prime}\right) S_{t}^{N, R}\left(d x^{\prime}, d v^{\prime}\right) d v=0 .
$$

However this is true due to

$$
\int_{\mathbb{R}^{2}} \theta^{1, \varepsilon_{N}}\left(v-v^{\prime}\right)\left(v-v^{\prime}\right) d v=0
$$

by the hypothesis of symmetry (3) in 2.3. The second identity of the Lemma follows by the very definition of $\delta_{X_{t}^{i, N, R}}^{\varepsilon_{N}}$. This ends the proof.

As stated above, we look for an estimate in $H^{2}\left(\mathbb{T}^{2}\right)$ for $u^{N}$. This is obtained by energy type estimates for the fluid in the vorticity form.

Lemma 5.4.

$$
\begin{aligned}
& \mathbf{E}\left[\sup _{t \in[0, T]}\left\|\omega_{t}^{N, R}\right\|_{L^{2}\left(\mathbb{T}^{2}\right)}^{2}+\int_{0}^{T}\left\|\nabla \omega_{s}^{N, R}\right\|_{L^{2}\left(\mathbb{T}^{2}\right)}^{2} d s\right] \lesssim \mathbf{E}\left[\left\|\omega_{0}^{N, R}\right\|_{L^{2}\left(\mathbb{T}^{2}\right)}^{2}\right] \\
& +\mathbf{E}\left[\left\|m_{1} F^{N, R}\right\|_{L^{2}\left([0, T] \times \mathbb{T}^{2}\right)}^{2}\right]+R \mathbf{E}\left[\left\|m_{0} F^{N, R}\right\|_{L^{2}\left([0, T] \times \mathbb{T}^{2}\right)}^{2}\right] .
\end{aligned}
$$

Proof. The thesis follows by classical energy inequality for $\omega^{N, R}$ and by using lemma 5.3.

We remark that the previous computation was only possible due to the presence of the cuf-off. The truncation is needed to decouple the fluid and particles in $\left(P S^{R}-N S^{R}\right)$, hence allowing us to close estimates for fluid and particles separately.

From Lemma 5.4 it is clear that it is necessary to control the $L^{2}$ norm of both $m_{1} F^{N, R}$ and $m_{0} F^{N, R}$. To do so we will exploit Lemma 3.5 and thus look for an estimate for $M_{6} F^{N, R}$ and for $\left(F^{N, R}\right)^{4}$. This is exactly the goal of the next lemmas.

Lemma 5.5. There exists a constant $C_{T, R, 4}$, independent on $N$, such that

$$
\sup _{t \in[0, T]} \mathbf{E}\left[\left\|F_{t}^{N, R}\right\|_{L^{4}\left(\mathbb{T}^{2} \times \mathbb{R}^{2}\right)}^{4}\right] \leq C_{T, R, 4}
$$

Proof. This proof strictly follows the proof of Lemma 3.3 in [14]. By Itô formula and integration by parts we have

$$
\begin{aligned}
\frac{1}{4} d & \int_{\mathbb{R}^{2}} \int_{\mathbb{T}^{2}}\left(F_{t}^{N, R}\right)^{4} d x d v+\frac{3 \sigma^{2}}{2} \int_{\mathbb{R}^{2}} \int_{\mathbb{T}^{2}}\left(F_{t}^{N, R}\right)^{2}\left|\nabla_{v} F_{t}^{N, R}\right|^{2} d x d v d t \\
= & -\int_{\mathbb{R}^{2}} \int_{\mathbb{T}^{2}}\left(F_{t}^{N, R}\right)^{3} \operatorname{div}_{x}\left(\theta^{\varepsilon_{N}} *\left(v S_{t}^{N, R}\right)\right) d x d v d t \\
& -\int_{\mathbb{R}^{2}} \int_{\mathbb{T}^{2}}\left(F_{t}^{N, R}\right)^{3} \operatorname{div}_{v}\left(\theta^{\varepsilon_{N}} *\left(u_{\varepsilon_{N}}^{N, R}(t, x) \chi_{R}\left(u_{t}^{N, R}\right)-v\right) S_{t}^{N, R}\right) d x d v d t \\
& +\int_{\mathbb{R}^{2}} \int_{\mathbb{T}^{2}}\left(F_{t}^{N, R}\right)^{3} d M_{t}^{N, \varepsilon_{N}} d x d v+\int_{\mathbb{R}^{2}} \int_{\mathbb{T}^{2}}\left(F_{t}^{N, R}\right)^{2} d\left[M^{N, \varepsilon_{N}}\right]_{t} d x d v .
\end{aligned}
$$


We estimate each of the terms above separately. Concerning (11), we can rewrite the convolution inside the integral as

$$
\operatorname{div}_{x}\left(\theta^{\varepsilon_{N}} *\left(v S_{t}^{N, R}\right)\right)=v \cdot \nabla_{x}\left(\theta^{\varepsilon_{N}} * S_{t}^{N, R}\right)-\left(\left(\nabla_{x} \theta^{\varepsilon_{N}} \cdot v\right) * S_{t}^{N, R}\right) .
$$

Hence, for the first term on the r.h.s. we have

$$
-\int_{\mathbb{R}^{2}} \int_{\mathbb{T}^{2}}\left(F_{t}^{N, R}\right)^{3} \nabla_{x} F_{t}^{N, R} \cdot v d x d v d t=\int_{\mathbb{R}^{2}} \int_{\mathbb{T}^{2}} \nabla_{x}\left(F_{t}^{N, R}\right)^{4} \cdot v d x d v d t=0 .
$$

For the second one, note that due to our hypothesis on the mollifiers $\theta^{0}(x)$ and $\theta^{1}(v)$ we have

$$
\begin{aligned}
& \left|\nabla_{x} \theta^{0, \varepsilon_{N}}\left(x-x^{\prime}\right)\right| \theta^{1, \varepsilon_{N}}\left(v-v^{\prime}\right)\left|\left(v-v^{\prime}\right)\right| \\
& \quad=\varepsilon_{N}^{-1} \varepsilon_{N}^{-d}\left|\nabla_{x} \theta^{0}\left(\varepsilon_{N}^{-1}\left(x-x^{\prime}\right)\right)\right| \varepsilon_{N}^{-d} \theta^{1}\left(\varepsilon_{N}^{-1}\left(v-v^{\prime}\right)\right)\left|v-v^{\prime}\right| \\
& \quad \leq \varepsilon_{N}^{-d}\left|\theta^{0}\left(\varepsilon_{N}^{-1}\left(x-x^{\prime}\right)\right)\right| \varepsilon_{N}^{-d} \theta^{1}\left(\varepsilon_{N}^{-1}\left(v-v^{\prime}\right)\right) \frac{\left|v-v^{\prime}\right|}{\varepsilon_{N}} \\
& \quad \leq \theta^{0, \varepsilon_{N}}\left(x-x^{\prime}\right) \theta^{1, \varepsilon_{N}}\left(v-v^{\prime}\right) 2
\end{aligned}
$$

implying

$$
|(11)| \lesssim\left\|F_{t}^{N, R}\right\|_{L^{4}\left(\mathbb{R}^{2} \times \mathbb{T}^{2}\right)}^{4}
$$

The main differences with respect to the proof of [14] concerns the term (12): we split it into two parts. One contains the fluid velocity $u$ and the other one contains the velocity variable: the first one follows easily by the truncation, being

$$
\begin{aligned}
\mid \int_{\mathbb{R}^{2}} & \int_{\mathbb{T}^{2}}\left(F_{t}^{N, R}\right)^{3} \operatorname{div}_{v}\left(\theta^{\varepsilon_{N}} * u_{\varepsilon_{N}}^{N, R}(t, x) \chi_{R}\left(u_{t}^{N, R}\right) S_{t}^{N, R}\right) d x d v \mid \\
= & \left|\int_{\mathbb{R}^{2}} \int_{\mathbb{T}^{2}} \nabla_{v}\left(F_{t}^{N, R}\right)^{3}\left(\theta^{\varepsilon_{N}} * u_{\varepsilon_{N}}^{N, R}(t, x) \chi_{R}\left(u_{t}^{N, R}\right) S_{t}^{N, R}\right) d x d v\right| \\
\leq & \int_{\mathbb{R}^{2}} \int_{\mathbb{T}^{2}}\left|\nabla_{v}\left(F_{t}^{N, R}\right)^{3}\right|\left(\theta^{\varepsilon_{N}} *\left|u_{\varepsilon_{N}}^{N, R}(t, x) \chi_{R}\left(u_{t}^{N, R}\right)\right| S_{t}^{N, R}\right) d x d v \\
\leq & R \int_{\mathbb{R}^{2}} \int_{\mathbb{T}^{2}}\left|\nabla_{v} F_{t}^{N, R} F_{t}^{N, R}\right|\left(F_{t}^{N, R}\right)^{2} d x d v \lesssim \frac{1}{\delta}|| F_{t}^{N, R}||_{L^{4}\left(\mathbb{T}^{2} \times \mathbb{R}^{2}\right)}^{4} \\
& +\delta \int_{\mathbb{R}^{2}} \int_{\mathbb{T}^{2}}\left(F_{t}^{N, R}\right)^{2}\left|\nabla_{v} F_{t}^{N, R}\right|^{2} d x d v
\end{aligned}
$$

and by choosing $\delta$ small enough we can take the second term to the l.h.s. maintaining the positivity. For the other one we again split it into a basic term plus a commutator

$$
\begin{aligned}
\int_{\mathbb{R}^{2}} & \int_{\mathbb{T}^{2}}\left(F_{t}^{N, R}\right)^{3} \operatorname{div}_{v}\left(\theta^{\varepsilon_{N}} * v S_{t}^{N, R}\right) d x d v \\
= & \int_{\mathbb{R}^{2}} \int_{\mathbb{T}^{2}}\left(F_{t}^{N, R}\right)^{3} \operatorname{div}_{v}\left(v\left(\theta^{\varepsilon_{N}} * S_{t}^{N, R}\right)\right) d x d v \\
& -\int_{\mathbb{R}^{2}} \int_{\mathbb{T}^{2}}\left(F_{t}^{N, R}\right)^{3} \operatorname{div}_{v}\left(\theta^{\varepsilon_{N}} v * S_{t}^{N, R}\right) d x d v
\end{aligned}
$$

The first term on the r.h.s. on (14) is easily handled by direct computation

$$
\begin{aligned}
= & -\int_{\mathbb{R}^{2}} \int_{\mathbb{T}^{2}} \nabla_{v}\left(F_{t}^{N, R}\right)^{3} \cdot v F_{t}^{N, R} d x d v= \\
& -\frac{1}{4} \int_{\mathbb{R}^{2}} \int_{\mathbb{T}^{2}} \nabla_{v}\left(F_{t}^{N, R}\right)^{4} \cdot v d x d v=\frac{1}{2}\left\|F_{t}^{N, R}\right\|_{L^{4}\left(\mathbb{T}^{2} \times \mathbb{R}^{2}\right)}^{4},
\end{aligned}
$$


while the second one is more tricky: we compute the divergence on $v$ and obtain

$$
\begin{aligned}
& \int_{\mathbb{R}^{2}} \int_{\mathbb{T}^{2}}\left(F_{t}^{N, R}\right)^{3} \operatorname{div}_{v}\left(\theta^{\varepsilon_{N}} v * S_{t}^{N, R}\right) d x d v=2 \int_{\mathbb{R}^{2}} \int_{\mathbb{T}^{2}}\left(F_{t}^{N, R}\right)^{4} d x d v \\
& \int_{\mathbb{R}^{2}} \int_{\mathbb{T}^{2}}\left(F^{N, R}\right)^{3} \int_{\mathbb{R}^{2}} \int_{\mathbb{T}^{2}} \theta^{0, \varepsilon_{N}}\left(x-x^{\prime}\right) \nabla_{v} \theta^{1, \varepsilon_{N}}\left(v-v^{\prime}\right) \cdot\left(v-v^{\prime}\right) S_{t}^{N, R}\left(d x^{\prime}, d v^{\prime}\right) d x d v d t \\
& \leq 2\left\|F_{t}^{N, R} \mid\right\|_{L^{4}\left(\mathbb{T}^{2} \times \mathbb{R}^{2}\right)}^{4} \\
& \quad+\int_{\mathbb{R}^{2}} \int_{\mathbb{T}^{2}}\left|\nabla\left(F^{N, R}\right)^{3}\right| \int_{\mathbb{R}^{2}} \int_{\mathbb{T}^{2}} \theta^{0, \varepsilon_{N}}\left(x-x^{\prime}\right) \theta^{1, \varepsilon_{N}}\left(v-v^{\prime}\right)\left|v-v^{\prime}\right| S_{t}^{N, R}\left(d x^{\prime}, d v^{\prime}\right) d x d v d t .
\end{aligned}
$$

Now we just look at the most inner term in the last inequality: using the compact support assumption for $\theta^{1}(v)$, see 2.3 hypothesis $(3)$, we get

$$
\theta^{0, \varepsilon_{N}}\left(x-x^{\prime}\right) \theta^{1, \varepsilon_{N}}\left(v-v^{\prime}\right)\left|v-v^{\prime}\right| \leq 2 \varepsilon_{N} \theta^{0, \varepsilon_{N}}\left(x-x^{\prime}\right) \theta^{1, \varepsilon_{N}}\left(v-v^{\prime}\right),
$$

which leads to (14) being

$$
\begin{aligned}
(14) & \lesssim\left\|F_{t}^{N, R}\right\|_{L^{4}\left(\mathbb{T}^{2} \times \mathbb{R}^{2}\right)}^{4}+\varepsilon_{N} \int_{\mathbb{R}^{2}} \int_{\mathbb{T}^{2}}\left|\nabla\left(F_{t}^{N, R}\right)^{3}\right| F_{t}^{N, R} d x d v \\
& \lesssim\left\|F_{t}^{N, R}\right\|_{L^{4}\left(\mathbb{T}^{2} \times \mathbb{R}^{2}\right)}^{4}+2 \varepsilon_{N} \int_{\mathbb{R}^{2}} \int_{\mathbb{T}^{2}}\left|\nabla_{v} F_{t}^{N, R}\right|^{2}\left(F_{t}^{N, R}\right)^{2} d x d v .
\end{aligned}
$$

We now deal with the two last term in (13): the integral with respect to the martingale $M_{t}^{N, \varepsilon_{N}}$ vanishes when computing the expected value, while for the integral with respect to the quadratic variation we have

$$
\begin{array}{r}
\int_{\mathbb{R}^{2}} \int_{\Pi^{d}}\left(F_{t}^{N}\right)^{2} d\left[M^{N, \varepsilon_{N}}\right]_{t} d x d v=\frac{\sigma^{2}}{N} \int_{\mathbb{R}^{2}} \int_{\Pi^{d}}\left(F_{t}^{N}\right)^{2}\left(\left|\nabla_{v} \theta^{\varepsilon_{N}}\right|^{2} * S_{t}^{N}\right) d x d v d t \leq \\
\sigma^{4}\left\|F_{t}^{N}\right\|_{L^{4}}^{4} d t+\frac{1}{N^{2}} \int_{\mathbb{R}^{2}} \int_{\Pi^{d}}\left(\left|\nabla_{v} \theta^{\varepsilon_{N}}\right|^{2} * S_{t}^{N}\right)^{2} d x d v d t .
\end{array}
$$

The square outside the convolution $\left(\left|\nabla_{v} \theta^{\varepsilon_{N}}\right|^{2} * S_{t}^{N}\right)^{2}$ can be troublesome, but we can handle it using the property of compact support of $\theta^{1}(v)$ and the separation of variables, in the following way:

$$
\begin{aligned}
& \int_{\mathbb{R}^{2}} \int_{\mathbb{T}^{2}}\left(\left|\nabla_{v} \theta^{\varepsilon_{N}}\right|^{2} * S_{t}^{N}\right)^{2} d x d v \\
& \quad \lesssim \frac{1}{N} \sum_{i=1}^{N}\left(\int_{\mathbb{R}^{2}} \int_{\mathbb{T}^{2}}\left|\nabla_{v} \theta^{1, \varepsilon_{N}}\left(v-V_{t}^{i, N, R}\right)\right|^{2} \theta^{0, \varepsilon_{N}}\left(x-X_{t}^{i, N, R}\right)^{2} d x d v\right)^{2} \\
& \quad \lesssim \frac{1}{N} \sum_{i=1}^{N} \int_{\mathbb{R}^{2}}\left|\nabla_{v} \theta^{1, \varepsilon_{N}}\left(v-V_{t}^{i, N, R}\right)\right|^{4} d v \int_{\mathbb{T}^{2}} \theta^{0, \varepsilon_{N}}\left(x-X_{t}^{i, N, R}\right)^{4} d x
\end{aligned}
$$

Now we compute

$$
\begin{aligned}
& \int_{\mathbb{R}^{2}}\left|\nabla_{v} \theta^{1, \varepsilon_{N}}\left(v-V_{t}^{i, N}\right)\right|^{4} d v=C N^{5 \beta}, \\
& \int_{\Pi^{d}} \theta^{0, \varepsilon_{N}}\left(x-X_{t}^{i, N}\right)^{4} d x=C N^{3 \beta},
\end{aligned}
$$

e substitute into the integral for the quadratic variation

$$
\frac{1}{N^{2}} \int_{\mathbb{R}^{2}} \int_{\Pi^{d}}\left(\left|\nabla_{v} \theta^{\varepsilon_{N}}\right|^{2} * S_{t}^{N}\right)^{2} d x d v \lesssim \frac{1}{N^{2}} N^{5 \beta} N^{3 \beta}
$$

which is bounded for $\beta \leq \frac{1}{4}$. 
Summarizing we have obtained

$$
\begin{aligned}
& d\left\|F_{t}^{N, R}\right\|_{L^{4}\left(\mathbb{T}^{2} \times \mathbb{R}^{2}\right)}^{4}+C \int_{\mathbb{R}^{2}} \int_{\mathbb{T}^{2}}\left(F_{t}^{N, R}\right)^{2}\left|\nabla_{v} F_{t}^{N, R}\right|^{2} d x d v d t \leq \\
& \quad \lesssim C_{R}\left\|F_{t}^{N, R}\right\|_{L^{4}\left(\mathbb{T}^{2} \times \mathbb{R}^{2}\right)}^{4} d t+\int_{\mathbb{R}^{2}} \int_{\mathbb{T}^{2}}\left(F_{t}^{N, R}\right)^{3} d M_{t}^{N, \varepsilon_{N}} d x d v+C d t
\end{aligned}
$$

which, after taking the average, ends the proof by standard Gronwall lemma.

By interpolation between $L^{p}$ spaces, and the fact that $F_{t}^{N, R}$ is a probability density function, we obtain the following corollary:

Corollary 5.6. There exists a constant $C_{T, R, 2}$, independent on $N$, such that

$$
\sup _{t \in[0, T]} \mathbf{E}\left[\left\|F_{t}^{N, R}\right\|_{L^{2}\left(\mathbb{T}^{2} \times \mathbb{R}^{2}\right)}^{2}\right] \leq C_{T, R, 2}
$$

We now proceed to bound the moments on the $v$-component of the mollified empirical measure $F^{N, R}$. The proof of the next Lemma follows by the very definition of $M_{k} F^{N, R}$ by using change of variable formula.

Lemma 5.7. For all $k \leq 6$ and for all $N$ and $R$, there exists a constant $C_{k}^{T, R}$, independent on $N$ such that

$$
\mathbf{E}\left[\sup _{t \in[0, T]} M_{k} F_{t}^{N, R}\right] \leq C_{k}^{T, R} .
$$

Proof. The proof follows by expanding $F^{N, R}$ as a summation, and by a change of variables inside the integral with respect to $v$. This allow to bound the $k$-th moments along $v$ of $F^{N, R}$ by

$$
\mathbf{E}\left[\sup _{t \in[0, T]}\left|V_{t}^{i, N, R}\right|^{k}\right] \text {. }
$$

Moreover, we can bound the expected value in the previous formula using the SDEs for the particles velocity, by using the truncation and the hypothesis on the initial conditions.

Summarizing, up to this point we were able to prove the following bounds, independently on $N$ :

$$
\begin{aligned}
& \sup _{t \in[0, T]} \mathbf{E}\left[\left\|m_{0} F_{t}^{N, R}\right\|_{L^{2}\left(\mathbb{T}^{2}\right)}^{2}\right] \leq C_{T, R}, \\
& \sup _{t \in[0, T]} \mathbf{E}\left[\left\|m_{1} F_{t}^{N, R}\right\|_{L^{2}\left(\mathbb{T}^{2}\right)}^{2}\right] \leq C_{T, R},
\end{aligned}
$$

by Lemmas 5.5, 5.7 and inequality 3. and 4. from Lemma 3.5. Also

$$
\mathbf{E}\left[\sup _{t \in[0, T]}\left\|\omega_{t}^{N, R}\right\|_{L^{2}\left(\mathbb{T}^{2}\right)}^{2}+\int_{0}^{T}\left\|\nabla \omega_{s}^{N, R}\right\|_{L^{2}\left(\mathbb{T}^{2}\right)}^{2} d s\right] \leq C_{T, R} .
$$

by Lemma 5.4 .

Hence we have obtained the desired bound for the fluid in vorticity form. However, in order to obtain convergence, we need to apply an appropriate tightness criterion.

Classical Aubin-Lions Lemma states that when $E_{0} \subseteq E \subseteq E_{1}$ are three Banach spaces with continuous embedding, and $E_{0}$ compactly embedded into $E$, then for all $p, q<\infty$ the space $L^{p}\left([0, T] ; E_{0}\right) \cap$ $W^{1, q}\left([0, T] ; E_{1}\right)$ is compactly embedded into $L^{p}([0, T] ; E)$. Hence, we can apply this criterion choosing $p=q=2$ and $E_{0}=H^{2}\left(\mathbb{T}^{2}\right), E=C\left(\mathbb{T}^{2}\right)$ and $E_{1}=H^{-1}\left(\mathbb{T}^{2}\right)$ to obtain

$$
L^{2}\left([0, T] ; H^{2}\left(\mathbb{T}^{2}\right)\right) \cap W^{1,2}\left([0, T] ; H^{-1}\left(\mathbb{T}^{2}\right)\right) \hookrightarrow L^{2}\left([0, T] ; C\left(\mathbb{T}^{2}\right)\right)
$$

and the embedding is compact. Thus, in order to obtain the required tightness result, we also need an a priori estimate for the time derivative of $\omega^{N, R}$ : 
Lemma 5.8. For every $\varepsilon>0$ there exists $Z>0$, such that

$$
\mathbf{P}\left(\left\|\omega^{N, R}\right\|_{W^{1,2}\left([0, T] ; H^{-1}\left(\mathbb{T}^{2}\right)\right)}>Z\right) \leq \varepsilon
$$

Proof. By Lemma 5.4 we already have the result for the $L^{2}\left([0, T] ; L^{2}\left(\mathbb{T}^{2}\right)\right)$ norm of $\omega^{N}$. Since $H^{1} \hookrightarrow$ $L^{2} \hookrightarrow H^{-1}$ we already know that

$$
\mathbf{P}\left(\left\|\omega^{N, R}\right\|_{L^{2}\left([0, T] ; H^{-1}\left(\mathbb{T}^{2}\right)\right)}>Z\right) \leq \varepsilon .
$$

Hence we only need to estimate $\left\|\partial_{t} \omega^{N, R}\right\|_{L^{2}\left([0, T] ; H^{-1}\left(\mathbb{T}^{2}\right)\right)}$. Thus we compute the $H^{-1}$ norm both sides in the equation for $\omega^{N, R}$, obtaining

$$
\begin{aligned}
& \left\|\partial_{t} \omega_{t}^{N, R}\right\|_{H^{-1}\left(\mathbb{T}^{2}\right)} \lesssim\left\|\Delta \omega_{t}^{N, R}\right\|_{H^{-1}\left(\mathbb{T}^{2}\right)}+\left\|u_{t}^{N, R} \cdot \nabla \omega_{t}^{N, R}\right\|_{H^{-1}\left(\mathbb{T}^{2}\right)} \\
& \quad+R\left\|m_{0} F_{t}^{N, R}\right\|_{L^{2}\left(\mathbb{T}^{2}\right)}+\left\|m_{1} F_{t}^{N, R}\right\|_{L^{2}\left(\mathbb{T}^{2}\right)}
\end{aligned}
$$

by classical argument and integration by parts. Taking the square and integrating both sides we obtain

$$
\begin{aligned}
& \int_{0}^{T}\left\|\partial_{t} \omega_{t}^{N, R}\right\|_{H^{-1}\left(\mathbb{T}^{2}\right)}^{2} d t \lesssim \int_{0}^{T}\left\|\nabla \omega_{t}^{N, R}\right\|_{L^{2}\left(\mathbb{T}^{2}\right)}^{2} d t \\
& \quad+\sup _{t \in[0, T]}\left\|\omega_{t}^{N, R}\right\|_{L^{2}\left(\mathbb{T}^{2}\right)}^{2} \int_{0}^{T}\left\|u_{t}^{N, R}\right\|_{C\left(\mathbb{T}^{2}\right)}^{2} d t \\
& \quad+R \int_{0}^{T}\left\|m_{0} F_{t}^{N, R}\right\|_{L^{2}\left(\mathbb{T}^{2}\right)}^{2} d t+\int_{0}^{T}\left\|m_{1} F_{t}^{N, R}\right\|_{L^{2}\left(\mathbb{T}^{2}\right)}^{2} d t .
\end{aligned}
$$

Finally, We compute probability both sides

$$
\mathbf{P}\left(\int_{0}^{T}\left\|\partial_{t} \omega_{t}^{N, R}\right\|_{H^{-1}\left(\mathbb{T}^{2}\right)}^{2} d t>R\right)
$$

and use the fact that we can split product term inside probabilities

$$
\begin{aligned}
& \mathbf{P}\left(\sup _{t \in[0, T]}\left\|\omega_{t}^{N, R}\right\|_{L^{2}\left(\mathbb{T}^{2}\right)}^{2} \int_{0}^{T}\left\|u_{t}^{N, R}\right\|_{C\left(\mathbb{T}^{2}\right)}^{2} d t>R\right) \\
& \quad \leq \mathbf{P}\left(\sup _{t \in[0, T]}\left\|\omega_{t}^{N, R}\right\|_{L^{2}\left(\mathbb{T}^{2}\right)}^{2}>\sqrt{R}\right)+\mathbf{P}\left(\int_{0}^{T}\left\|u_{t}^{N, R}\right\|_{C\left(\mathbb{T}^{2}\right)}^{2} d t>\sqrt{R}\right) .
\end{aligned}
$$

Since all the terms above are bounded in expected value, we can apply Chebyshev inequality to make each term smaller than $\varepsilon$. This ends the proof.

At this point, thanks to Aubin's Lemma, we are able to obtain a first tightness result for the law of $u^{N, R}$ in $L^{2}\left([0, T] ; C\left(\mathbb{T}^{2}\right)\right) . L^{2}$ estimates on time are enough to prove a convergence result (as partially done in [14]), but they are not sufficient to remove the cutoff at the particle level, thus obtaining Theorem 2.3. Hence we will have to improve our estimates in order to obtain stronger time convergence. We apply Corollary 8 in [25] by taking

$$
X=H^{1+2 \alpha}\left(\mathbb{T}^{2}\right), \quad B=H^{1+2 \alpha-\varepsilon}\left(\mathbb{T}^{2}\right), \quad Y=H^{-1}\left(\mathbb{T}^{2}\right),
$$

where $\varepsilon<2 \alpha$ and where $X \hookrightarrow Y$ is compact. The interpolation inequality between the space $B$ and $X, Y$, required in Corollary 8 , it is an easy result of Fourier analysis since we are on the torus. Hence we have that

$$
L^{\infty}\left([0, T] ; H^{1+2 \alpha}\left(\mathbb{T}^{2}\right)\right) \cap W^{1,2}\left([0, T] ; H^{-1}\left(\mathbb{T}^{2}\right)\right) \hookrightarrow C\left([0, T] ; H^{1+2 \alpha-\varepsilon}\left(\mathbb{T}^{2}\right)\right)
$$

with a compact embedding. Hence, by Sobolev embedding in dimension two of $H^{1+2 \alpha-\varepsilon}\left(\mathbb{T}^{2}\right)$ into $C\left(\mathbb{T}^{2}\right)$ we also have that

$$
L^{\infty}\left([0, T] ; H^{1+2 \alpha}\left(\mathbb{T}^{2}\right)\right) \cap W^{1,2}\left([0, T] ; H^{-1}\left(\mathbb{T}^{2}\right)\right) \hookrightarrow C\left([0, T] \times \mathbb{T}^{2}\right)
$$


with a compact embedding. Clearly the result also holds when $H^{1+2 \alpha}\left(\mathbb{T}^{2}\right)$ is replaced by $H^{2}\left(\mathbb{T}^{2}\right)$. However we were not able to obtain a uniform in time result for the $H^{2}$ norm and hence we tried to trim our requirements. To do so, we first rewrite the equation for $\omega^{N, R}$ in its mild formulation

$$
\begin{aligned}
& \omega_{t}^{N, R}=e^{t \Delta} \omega_{0}^{N, R}-\int_{0}^{t} e^{(t-s) \Delta} u_{s}^{N, R} \cdot \nabla \omega_{s}^{N, R} d s \\
& -\int_{0}^{t} e^{(t-s) \Delta} \nabla^{\perp} \cdot \frac{1}{N} \sum_{i=1}^{N}\left(u_{\varepsilon_{N}}^{N, R}\left(X_{s}^{i, N, R}\right) \chi_{R}\left(u_{s}^{N, R}\right)-V_{s}^{i, N, R}\right) \delta_{X_{s}^{i, N, R}}^{\varepsilon_{N}} d s .
\end{aligned}
$$

Lemma 5.9. For all $\alpha<\frac{1}{2}$ and for each $\varepsilon$, there exists $Z$ such that

$$
\mathbf{P}\left(\left\|u^{N, R}\right\|_{L^{\infty}\left([0, T], H^{1+2 \alpha}\right)}>Z\right) \leq \varepsilon
$$

Proof. We apply a generalized Gronwall Lemma to the function of the only time variable $\left\|u_{t}^{N, R}\right\|_{H^{1+2 \alpha}\left(\mathbb{T}^{2}\right)}$. Since $\left\|u_{t}^{N, R}\right\|_{H^{1+2 \alpha}\left(\mathbb{T}^{2}\right)} \sim\left\|\omega_{t}^{N, R}\right\|_{H^{2 \alpha}\left(\mathbb{T}^{2}\right)}$ we apply the operator $(I-\Delta)^{\alpha}$ on the mild formulation of vorticity equation (15), obtaining

$$
\begin{aligned}
& \left\|(I-\Delta)^{\alpha} \omega_{t}^{N, R}\right\|_{L^{2}\left(\mathbb{T}^{2}\right)} \leq\left\|(I-\Delta)^{\alpha} e^{t \Delta} \omega_{0}^{N, R}\right\|_{L^{2}\left(\mathbb{T}^{2}\right)} \\
& \quad+\int_{0}^{t}\left\|(I-\Delta)^{\alpha} e^{(t-s) \Delta} \nabla^{\perp} \cdot \frac{1}{N} \sum_{i=1}^{N}\left(u_{\varepsilon_{N}^{N, R}}^{N}\left(X_{s}^{i, N, R}\right) \chi_{R}\left(u_{s}^{N, R}\right)-V_{s}^{i, N, R}\right) \delta_{X_{s}^{i, N, R}}^{\varepsilon_{N}}\right\|_{L^{2}\left(\mathbb{T}^{2}\right)} \\
& \quad+\int_{0}^{t}\left\|(I-\Delta)^{\alpha} e^{(t-s) \Delta} u_{s}^{N, R} \cdot \nabla \omega_{s}^{N, R}\right\|_{L^{2}\left(\mathbb{T}^{2}\right)} d s .
\end{aligned}
$$

We start by estimating the initial conditions:

$$
\begin{aligned}
& \left\|(I-\Delta)^{\alpha} \omega_{0}^{N, R}\right\|_{L^{2}\left(\mathbb{T}^{2}\right)}\left\|(I-\Delta)^{\alpha} \omega_{0}^{N, R}\right\|_{L^{2}\left(\mathbb{T}^{2}\right)} \lesssim\left\|\omega_{0}^{N, R}\right\|_{H^{2 \alpha}\left(\mathbb{T}^{2}\right)} .
\end{aligned}
$$

Regarding the second term of the r.h.s. of (16)

$$
\begin{aligned}
\| & (I-\Delta)^{\alpha} e^{(t-s) \Delta} \nabla^{\perp} \cdot \frac{1}{N} \sum_{i=1}^{N}\left(u_{\varepsilon_{N}}^{N, R}\left(X_{s}^{i, N, R}\right) \chi_{R}\left(u_{s}^{N, R}\right)-V_{s}^{i, N, R}\right) \delta_{X_{s}^{i, N, R}}^{\varepsilon_{N}} \|_{L^{2}\left(\mathbb{T}^{2}\right)} \\
\leq & \left\|\nabla(I-\Delta)^{-1 / 2}\right\|_{L^{2}\left(\mathbb{T}^{2}\right) \rightarrow L^{2}\left(\mathbb{T}^{2}\right)}\left\|(I-\Delta)^{\alpha+1 / 2} e^{(t-s) \Delta}\right\|_{L^{2}\left(\mathbb{T}^{2}\right) \rightarrow L^{2}\left(\mathbb{T}^{2}\right)} \\
& \times\left\|\frac{1}{N} \sum_{i=1}^{N}\left(u_{\varepsilon_{N}}^{N, R}\left(X_{s}^{i, N, R}\right) \chi_{R}\left(u_{s}^{N, R}\right)-V_{s}^{i, N, R}\right) \delta_{X_{s}^{i, N, R}}^{\varepsilon_{N}}\right\|_{L^{2}\left(\mathbb{T}^{2}\right)} \\
\leq & \frac{C}{(t-s)^{\alpha+1 / 2}}\left\|\frac{1}{N} \sum_{i=1}^{N}\left(u_{\varepsilon_{N}}^{N, R}\left(X_{s}^{i, N, R}\right) \chi_{R}\left(u_{s}^{N, R}\right)-V_{s}^{i, N, R}\right) \delta_{X_{s}^{i, N, R}}^{\varepsilon_{N}}\right\|_{L^{2}\left(\mathbb{T}^{2}\right)} \\
\leq & \frac{C}{(t-s)^{\alpha+1 / 2}}\left(R\left\|m_{0} F_{t}^{N, R}\right\|_{L^{2}\left(\mathbb{T}^{2}\right)}+\left\|m_{1} F_{t}^{N, R}\right\|_{L^{2}\left(\mathbb{T}^{2}\right)}\right),
\end{aligned}
$$


while for the last one of (16) we have

$$
\begin{aligned}
& \left\|(I-\Delta)^{\alpha} e^{(t-s) \Delta} u_{s}^{N, R} \cdot \nabla \omega_{s}^{N, R}\right\|_{L^{2}\left(\mathbb{T}^{2}\right)}\left\|(I-\Delta)^{-1 / 2} u_{s}^{N, R} \cdot \nabla \omega_{s}^{N, R}\right\|_{L^{2}\left(\mathbb{T}^{2}\right)} \\
& \quad \leq\left\|(I-\Delta)^{\alpha+1 / 2} e^{(t-s) \Delta}\right\|_{L^{2}\left(\mathbb{T}^{2}\right) \rightarrow L^{2}\left(\mathbb{T}^{2}\right)} \|(I) \\
& \quad \leq \frac{C}{(t-s)^{\alpha+1 / 2}}\left\|(I-\Delta)^{-1 / 2} u_{s}^{N, R} \cdot \nabla \omega_{s}^{N, R}\right\|_{L^{2}\left(\mathbb{T}^{2}\right)},
\end{aligned}
$$

and

$$
\left\|(I-\Delta)^{-1 / 2} u_{s}^{N, R} \cdot \nabla \omega_{s}^{N, R}\right\|_{L^{2}\left(\mathbb{T}^{2}\right)}=\sup _{\varphi \in L^{2}\left(\mathbb{T}^{2}\right)}\left|\left\langle(I-\Delta)^{-1 / 2} u_{s}^{N, R} \cdot \nabla \omega_{s}^{N, R}, \varphi\right\rangle\right| .
$$

Now, notice that

$$
\begin{aligned}
& \left\langle u_{s}^{N, R} \cdot \nabla \omega_{s}^{N, R},(I-\Delta)^{-1 / 2} \varphi\right\rangle=-\left\langle\omega_{s}^{N, R}, u_{s}^{N, R} \cdot \nabla(I-\Delta)^{-1 / 2} \varphi\right\rangle \\
& \quad \leq \sup _{\|\varphi\|_{L^{2}\left(\mathbb{T}^{2}\right)} \leq 1}\|\varphi\|_{L^{2}\left(\mathbb{T}^{2}\right)}\left\|u_{s}^{N, R}\right\|_{\infty}\left\|\omega^{N, R}\right\|_{L^{2}\left(\mathbb{T}^{2}\right)} .
\end{aligned}
$$

Combining (17),(18),(19):

$$
\begin{aligned}
& \left\|\omega_{t}^{N, R}\right\|_{H^{2 \alpha}\left(\mathbb{T}^{2}\right)} \lesssim\left\|\omega_{0}^{N, R}\right\|_{H^{2 \alpha}\left(\mathbb{T}^{2}\right)} \\
& \quad+\int_{0}^{t} \frac{\left(R\left\|m_{0} F_{s}^{N, R}\right\|_{L^{2}\left(\mathbb{T}^{2}\right)}+\left\|m_{1} F_{s}^{N, R}\right\|_{L^{2}\left(\mathbb{T}^{2}\right)}\right)}{(t-s)^{\alpha+1 / 2}} d s \\
& \quad+\int_{0}^{t} \frac{\left\|u_{s}^{N, R}\right\|_{L^{\infty}\left(\mathbb{T}^{2}\right)}\left\|\omega_{s}^{N, R}\right\|_{L^{2}\left(\mathbb{T}^{2}\right)}}{(t-s)^{\alpha+1 / 2}} d s \\
& \leq C \\
& \quad+C \omega_{0}^{N, R} \|_{H^{2 \alpha}\left(\mathbb{T}^{2}\right)}+\int_{0}^{T} \frac{\left(R\left\|m_{0} F_{s}^{N, R}\right\|_{L^{2}\left(\mathbb{T}^{2}\right)}+\left\|m_{1} F_{s}^{N, R}\right\|_{L^{2}\left(\mathbb{T}^{2}\right)}\right)}{(T-s)^{\alpha+1 / 2}} d s+ \\
& \left.\quad\left\|\omega_{t}^{N, R}\right\|_{L^{2}\left(\mathbb{T}^{2}\right)}\right) \int_{0}^{t} \frac{\left\|u_{s}^{N, R}\right\|_{H^{1+2 \alpha}\left(\mathbb{T}^{2}\right)}}{(t-s)^{\alpha+1 / 2}} d s .
\end{aligned}
$$

Finally,

$$
\begin{aligned}
& \left\|u_{t}^{N, R}\right\|_{H^{1+2 \alpha}\left(\mathbb{T}^{2}\right)} \lesssim C\left\|\omega_{0}^{N, R}\right\|_{H^{2 \alpha}\left(\mathbb{T}^{2}\right)}+\int_{0}^{T} \frac{R\left\|m_{0} F_{s}^{N, R}\right\|_{L^{2}\left(\mathbb{T}^{2}\right)}}{(T-s)^{\alpha+1 / 2}} d s+ \\
& \quad+\int_{0}^{T} \frac{\left\|m_{1} F_{s}^{N, R}\right\|_{L^{2}\left(\mathbb{T}^{2}\right)}}{(T-s)^{\alpha+1 / 2}} d s+\left(\sup _{t \in[0, T]}\left\|\omega_{t}^{N, R}\right\|_{L^{2}\left(\mathbb{T}^{2}\right)}\right) \int_{0}^{t} \frac{\left\|u_{s}^{N, R}\right\|_{H^{1+2 \alpha}\left(\mathbb{T}^{2}\right)}}{(t-s)^{\alpha+1 / 2}} d s .
\end{aligned}
$$

Notice that in the expression above the terms involved above are random (for simplicity we have omitted $\omega \in \Omega$ ). Introduce, to short the notation, the random function

$$
\varphi(t):=\left\|u_{t}^{N, R}\right\|_{H^{1+2 \alpha}\left(\mathbb{T}^{2}\right)} .
$$

We have proved that the function $\varphi$ satisfies

$$
\varphi(t) \leq X_{1}+X_{2} \int_{0}^{t} \frac{\varphi(s)}{(t-s)^{\alpha+1 / 2}} d s
$$

where

$$
\begin{aligned}
& X_{1}=\left\|\omega_{0}^{N, R}\right\|_{H^{2 \alpha}\left(\mathbb{T}^{2}\right)}+\int_{0}^{T} \frac{\left(R\left\|m_{0} F_{s}^{N, R}\right\|_{L^{2}\left(\mathbb{T}^{2}\right)}+\left\|m_{1} F_{s}^{N, R}\right\|_{L^{2}\left(\mathbb{T}^{2}\right)}\right)}{(T-s)^{\alpha+1 / 2}} d s \\
& X_{2}=\sup _{t \in[0, T]}\left\|\omega_{t}^{N, R}\right\|_{L^{2}\left(\mathbb{T}^{2}\right)} .
\end{aligned}
$$


Notice that, by the uniform estimates proved in this section, there exist two constant $C_{1}$ and $C_{2}$, independent on $N$, such that

$$
\mathbf{E}\left[X_{1}\right] \leq C_{1}, \quad \mathbf{E}\left[X_{2}\right] \leq C_{2},
$$

so that, for fixed $\varepsilon$ we can chose $R_{1}, R_{2}>0$ in order to have

$$
\mathbf{P}\left(X_{1}>R_{1}\right)<\frac{\varepsilon}{2}, \quad \mathbf{P}\left(X_{2}>R_{2}\right)<\frac{\varepsilon}{2} .
$$

For a fixed $\omega \in \Omega$ applying Gronwall Lemma to the function $\varphi$ we obtain

$$
\sup _{t \in[0, T]} \varphi(t)(\omega) \leq f\left(X_{1}, X_{2}\right)(\omega)
$$

We now claim that

$$
\mathbf{P}\left(\sup _{t \in[0, T]} \varphi(t)>f\left(R_{1}, R_{2}\right)\right)<\varepsilon .
$$

In fact we have the following chain of inequalities

$$
\begin{aligned}
& \mathbf{P}\left(\sup _{t \in[0, T]} \varphi(t) \leq f\left(R_{1}, R_{2}\right)\right) \geq \mathbf{P}\left(\varphi(t) \leq R_{1}+R_{2} \int_{0}^{t} \frac{\varphi(s)}{(t-s)^{\alpha+1 / 2}} d s\right) \\
& \quad \geq \mathbf{P}\left(\left(X_{1} \leq R_{1}\right) \cap\left(X_{2} \leq R_{2}\right)\right) \geq 1-\mathbf{P}\left(X_{1}>R_{1}\right)-\mathbf{P}\left(X_{2}>R_{2}\right) \geq 1-\varepsilon .
\end{aligned}
$$

We end the proof by taking the complement set both sides.

We are finally able to obtain the following tightness result:

Lemma 5.10. The family of laws $\left\{Q^{N, R, u}\right\}_{N \in \mathbb{N}}$ of $\left\{u^{N, R}\right\}_{N \in \mathbb{N}}$, is tight, and hence is relatively compact as a probability measure on $C\left([0, T] \times \mathbb{T}^{2}\right)$.

Proof. The proof is just an application of Simons embedding in [25]. For each $M, Z>0$ we can consider the following set, for all $\alpha<1 / 2$

$$
\begin{aligned}
& K_{M, Z}=\left\{u \in C\left([0, T] \times \mathbb{T}^{2}\right) \mid\|u\|_{L^{\infty}\left([0, T] ; H^{1+2 \alpha}\left(\mathbb{T}^{2}\right)\right)} \leq M,\right. \\
& \left.\|u\|_{W^{1,2}\left([0, T] ; H^{-1}\left(\mathbb{T}^{2}\right)\right)} \leq Z\right\} .
\end{aligned}
$$

By the Simons Lemma $K_{M, Z}$ is relatively compact in $C\left([0, T] \times \mathbb{T}^{2}\right)$. Notice that

$$
\begin{aligned}
& Q^{N, R, u}\left(K_{M, Z}^{c}\right)=\mathbf{P}\left(u^{N, R} \in K_{M, Z}^{c}\right) \\
& \quad \leq \mathbf{P}\left(\left\|u^{N, R}\right\|_{L^{\infty}\left([0, T] ; H^{1+2 \alpha}\left(\mathbb{T}^{2}\right)\right)}>M\right)+\mathbf{P}\left(\left\|u^{N, R}\right\|_{W^{1,2}\left([0, T] ; H^{-1}\left(\mathbb{T}^{2}\right)\right)}>Z\right) \leq \\
& \quad \leq \frac{\mathbf{E}\left[\left\|u^{N, R}\right\|_{L^{\infty}\left([0, T] ; H^{1+2 \alpha}\left(\mathbb{T}^{2}\right)\right)}\right]}{M}+\varepsilon
\end{aligned}
$$

by lemma 5.8. By Lemma 5.9 the expected values on the r.h.s. is uniformly bounded with respect to $N$, hence the sequence $\left\{Q^{N, R, u}\right\}_{N \in \mathbb{N}}$ is tight and proof is concluded.

Combining Proposition 5.2 and Lemma 5.10 we obtain the following:

Corollary 5.11. The family of laws $\left\{Q^{N, R}\right\}_{N \in \mathbb{N}}$ of the couple $\left(u^{N, R}, S^{N, R}\right)$ is tight, and hence relatively compact as a probability measures on $C\left([0, T] \times \mathbb{T}^{2}\right) \times C\left([0, T] ; \mathbf{P}_{1}\left(\mathbb{T}^{2} \times \mathbb{R}^{2}\right)\right)$. 


\subsection{Convergence of $\left(P S^{R}-N S^{R}\right)$ to $(V N S)$}

We will now prove that, under hypothesis on Section 2.3, and if $R$ is large enough, then the solution $\left(u^{R}, F^{R}\right)$ of $\left(V N S^{R}\right)$ coincide with the solution $(u, F)$ of $(V N S)$. To do so we will prove that $u^{R}$ is bounded in $L^{\infty}\left([0, T] \times \mathbb{T}^{2}\right)$, independently on $R$. First we summarize all the intermediate results needed for the proof. We remark that all the following bounds hold independently on $R$ :

- For all $k \leq 6$

$$
\sup _{t \in[0, T]} M_{k} F_{t}^{R} \leq C
$$

by Lemma 3.6 and hypothesis 2.3 ;

$$
\left\|m_{0} F^{R}\right\|_{L^{\infty}\left([0, T] ; L^{2}\left(\mathbb{T}^{2}\right)\right.} \leq C, \quad \text { and } \quad\left\|m_{1} F^{R}\right\|_{L^{\infty}\left([0, T] ; L^{2}\left(\mathbb{T}^{2}\right)\right.} \leq C
$$

by Lemma 3.6 and inequality 1 . and 2 . in Lemma 3.5 ;

- for all $p>1$

$$
\left\|u^{R}\right\|_{L^{2}\left([0, T] ; L^{p}\left(\mathbb{T}^{2}\right)\right)} \leq C_{p}
$$

by Remark 3.2.

We can now formulate a further result, needed in the proof of Theorem 5.14.

Lemma 5.12. There exists a constant $C$, independent on $R$, such that

$$
\left\|\omega^{R}\right\|_{L^{\infty}\left([0, T] ; L^{2}\left(\mathbb{T}^{2}\right)\right)} \leq C .
$$

Proof. Computing the time derivative of $\int_{\mathbb{T}^{2}}\left|\omega_{t}^{R}\right|^{2} d x$ we obtain

$$
\begin{aligned}
& \left\|\omega_{t}^{R}\right\|_{L^{2}\left(\mathbb{T}^{2}\right)}^{2}+\int_{0}^{T} \int_{\mathbb{T}^{2}}\left|\nabla \omega_{s}^{R}\right|^{2} d x d s \lesssim\left\|\omega_{0}\right\|_{L^{2}\left(\mathbb{T}^{2}\right)}^{2} \\
& \quad+\int_{0}^{t} \int_{\mathbb{T}^{2}} \omega_{s}^{R} \nabla^{\perp} \cdot \int_{\mathbb{R}^{2}}\left(u_{s}^{R}-v\right) \chi_{R}\left(u^{R)} F_{s}^{R} d v d x d s .\right.
\end{aligned}
$$

Focusing only on the last term of the previous inequality we have

$$
\begin{aligned}
(20) \lesssim & \int_{0}^{t} \int_{\mathbb{T}^{2}}\left|\nabla \omega_{s}^{R}\right|\left|u_{s}^{R}\right| \int_{\mathbb{R}^{2}} F_{s}^{R} d v d x d s+\int_{0}^{t} \int_{\mathbb{T}^{2}}\left|\nabla \omega_{s}^{R}\right| \int_{\mathbb{R}^{2}}|v| F_{s}^{R} d v d x d s \\
\lesssim & \int_{0}^{T} \int_{\mathbb{T}^{2}}\left|\nabla \omega_{s}^{R}\right|^{2} d x d s+\int_{0}^{T} \int_{\mathbb{T}^{2}}\left|u_{s}^{R}\right|^{2}\left(\int_{\mathbb{R}^{2}} F_{s}^{R} d v\right)^{2} d x d s \\
& +\int_{0}^{T} \int_{\mathbb{T}^{2}}\left|\nabla \omega_{s}^{R}\right|^{2} d x d s+\int_{0}^{T} \int_{\mathbb{T}^{2}}\left(\int_{\mathbb{R}^{2}}|v| F_{s}^{R} d v\right)^{2} d x d s .
\end{aligned}
$$

Let us notice that

$$
\begin{aligned}
& \int_{0}^{T} \int_{\mathbb{T}^{2}}\left|u_{s}^{R}\right|^{2}\left(\int_{\mathbb{R}^{2}} F_{s}^{R} d v\right)^{2} d x d s \leq \int_{0}^{T}\left\|u_{s}^{R}\right\|_{L^{4}\left(\mathbb{T}^{2}\right)}^{2}\left(\int_{\mathbb{T}^{2}}\left(\int_{\mathbb{R}^{2}} F_{s}^{R} d v\right)^{4} d x\right)^{\frac{1}{2}} d s \\
& \quad \leq \sup _{t \in[0, T]}\left\|m_{0} F_{t}^{R}\right\|_{L^{4}\left(\mathbb{T}^{2}\right)}^{2}\left\|u^{R}\right\|_{L^{2}\left([0, T] ; L^{4}\left(\mathbb{T}^{2}\right)\right)} \\
& \quad \lesssim \sup _{t \in[0, T]}\left(M_{6} F_{t}^{R}\right)^{\frac{1}{2}}\left\|u^{R}\right\|_{L^{2}\left([0, T] ; L^{4}\left(\mathbb{T}^{2}\right)\right)} \leq C
\end{aligned}
$$

and

$$
\int_{0}^{T} \int_{\mathbb{T}^{2}}\left(\int_{\mathbb{R}^{2}}|v| F_{s}^{R} d v\right)^{2} d x d s \lesssim_{T} \sup _{t \in[0, T]} M_{4} F_{t}^{R} \leq C
$$

again by Lemma 3.5. We conclude the proof by classical Gronwall Lemma. 
We emphasize that, even if it is possible to prove the uniform bound with respect the parameter $R$, it is not possible to obtain the same result directly at the particle level. In other terms, we were not able to obtain directly any bound on the vorticity in the particle system $(P S-N S)$

$$
\mathbf{E}\left[\left\|\omega^{N}\right\|_{L^{\infty}\left([0, T] ; L^{2}\left(\mathbb{T}^{2}\right)\right)}\right]
$$

without using the cut off. This result would have allowed us to remove the cut off directly at the particle level, without any further complication.

We finally prove the uniform bound on $u^{R}$ :

Proposition 5.13. There exists a constant $\mathbf{K}_{u}$, independent on $R$, such that

$$
\left\|u^{R}\right\|_{\infty} \leq \mathbf{K}_{u}
$$

Proof. In order to produce the required bound we bound uniformly the norm of $u^{R}$ in the space $L^{\infty}\left([0, T] ; H^{1+2 \alpha}\left(\mathbb{T}^{2}\right)\right)$ for any $\alpha<1 / 2$. Hence we use the mild formulation for the vorticity equation associated with $u^{R}$ :

$$
\partial_{t} \omega^{R}=\Delta \omega^{R}-u^{R} \cdot \nabla \omega^{R}-\nabla^{\perp} \cdot \int_{\mathbb{R}^{2}}\left(u^{R}-v\right) \chi_{R}\left(u^{R)} F^{R} d v .\right.
$$

Following the same argument of Lemma 5.9 we get

$$
\begin{aligned}
& \left\|u_{t}^{R}\right\|_{H^{1+2 \alpha}\left(\mathbb{T}^{2}\right)} \lesssim\left\|\omega_{t}^{R}\right\|_{H^{2 \alpha}\left(\mathbb{T}^{2}\right)} \lesssim\left\|\omega_{0}^{R}\right\|_{H^{2 \alpha}\left(\mathbb{T}^{2}\right)} \\
& \quad+\int_{0}^{t} \frac{\left.\left\|u_{s}^{R}\right\|\right|_{L^{\infty}\left(\mathbb{T}^{2}\right)}\left\|\omega_{s}^{R}\right\|_{L^{2}\left(\mathbb{T}^{2}\right)}}{(t-s)^{\alpha+1 / 2}} d s \\
& \quad+\int_{0}^{t} \frac{\left.\left\|u_{s}^{R}\right\|\right|_{L^{\infty}\left(\mathbb{T}^{2}\right)}\left\|m_{0} F_{s}^{R}\right\|_{L^{2}\left(\mathbb{T}^{2}\right)}}{(t-s)^{\alpha+1 / 2}} d s+\int_{0}^{t} \frac{\left\|m_{1} F_{s}^{R}\right\|_{L^{2}\left(\mathbb{T}^{2}\right)}}{(t-s)^{\alpha+1 / 2}} d s \\
& \lesssim\left\|\omega_{0}^{R}\right\|_{H^{2 \alpha}\left(\mathbb{T}^{2}\right)}+\left\|\omega^{R}\right\|_{L^{\infty}\left([0, T] ; L^{2}\left(\mathbb{T}^{2}\right)\right)} \int_{0}^{t} \frac{\left\|u_{s}^{R}\right\|_{H^{1+2 \alpha}\left(\mathbb{T}^{2}\right)}}{(t-s)^{\alpha+1 / 2}} d s \\
& \quad+\left(\sup _{t \in[0, T]} M_{2} F_{t}^{R}\right)^{\frac{1}{2}} \int_{0}^{t} \frac{\left\|u_{s}^{R}\right\|_{H^{1+2 \alpha}\left(\mathbb{T}^{2}\right)}}{(t-s)^{\alpha+1 / 2}} d s+\left(\sup _{t \in[0, T]} M_{4} F_{t}^{R}\right)^{\frac{1}{2}},
\end{aligned}
$$

by neglecting the cutoff function $\chi_{R}$ which is bounded by one. By using the uniform bound described at the beginning of Sect. 5.2, Lemma 5.12 and Lemma 3.5 inequality 1. and 2. we see that all the expression above are bounded independently on $R$ and we conclude by a Gronwall type argument applied to the function $\left\|u_{t}^{R}\right\|_{H^{1+2 \alpha}\left(\mathbb{T}^{2}\right)}$.

In conclusion we have the following Theorem:

Theorem 5.14. If $R \geq \mathbf{K}_{u}+1$, then any weak solution $\left(u^{R}, F^{R}\right)$ of system of PDE $\left(V N S^{R}\right)$ coincide with the unique bounded weak solutions of system of equations (VNS).

Proof. By proposition 5.13, taking $R \geq \mathbf{K}_{u}+1$ we have that the function $\chi_{R}\left(u^{R}\right) \equiv 1$, hence system of equation $\left(V N S^{R}\right)$ reduce to $(V N S)$. Hence, we obtain that the couple $\left(u^{R}, F^{R}\right)$ satisfies system of equation $(V N S)$. By the uniqueness of solution for system of equations $(V N S)$, we obtain $u=u^{R}$ and $F=F^{R}$.

In order to complete the proof of Proposition 5.1 we need only to verify that limit points of the sequence $\left\{Q^{N, R}\right\}_{N \in \mathbb{N}}$ are supported on weak solutions of system of equations (VNS).

Proposition 5.15. If $R \geq \mathbf{K}_{u}+1$ limit points of subsequences of $\left\{Q^{N, R}\right\}_{N \in \mathbb{N}}$ are supported on the bounded weak solutions of system of PDE (VNS) (see Definition 2.2). 
Proof. In order to prove that weak limits are supported on weak solutions, we have to prove that those object satisfies equation $(V N S)$ in the weak sense, and that they have the correct regularity. The fact that limit objects satisfy system of equations $(V N S)$ is classical, see [23]. Let us focus on the regularity issue.

First, by By Lemma 5.4 together with Lemma 3.5 inequality 3. and 4, limit points on the component corresponding to $u^{N, R}$ satisfy the regularity properties of Definition 2.2. Similarly from a priori estimates in Corollary 5.6 limit points of subsequences have a density on their particle component (corresponding to $\left.S^{N, R}\right)$ which is also in $L^{2}\left([0, T] \times \mathbb{T}^{2} \times \mathbb{R}^{2}\right)$. In order to complete the proof we need to verify that such density is uniformly bounded, as required in Definition 2.2. This follows by the maximum principle argued in Sect. 3.1. Namely, the fact that the limit points along the particles component satisfies system of equations $(V N S)$, where $u$ is uniformly bounded, yields to an uniform bound for the density in $L^{\infty}\left([0, T] \times \mathbb{T}^{2} \times \mathbb{R}^{2}\right)$. Denoting by $F$ one of the limit points, we only need to verify that

$$
\int_{0}^{T} \int_{\mathbb{T}^{2}} \int_{\mathbb{R}^{2}}|v|^{2} F_{s}^{2} d x d v d s<\infty .
$$

By using Lemma $5.5 F$ is in $L^{4}\left([0, T] \times \mathbb{T}^{2} \times \mathbb{R}^{2}\right)$. By interpolation inequality of $L^{p}$ spaces we also have $F \in L^{3}\left([0, T] \times \mathbb{T}^{2} \times \mathbb{R}^{2}\right)$. Also, the uniform bound on the $v$-moments of $F^{N, R}$, provided in Lemma 5.7, grants also $M_{4} F$ to be finite. Hence, by an easy computation (see Section 3.1), we see that (21) is satisfied. Thus by the maximum principle we have $F \in L^{\infty}\left([0, T] \times \mathbb{T}^{2} \times \mathbb{R}^{2}\right)$, hence ending the proof.

Combining Proposition 5.15 with Theorem 4.1 we complete the proof of Proposition 5.1.

\section{Scaling Limit for the Full System}

The aim of this section is to prove that the cut-off can be removed also in the approximating system $\left(u^{N, R}, S^{N, R}\right)$ : the uniform convergence result proved in the previous section, Proposition 5.1, gives a simple but relevant hint to prove the final result of convergence. We expect that the converging object $\left(u^{N, R}, S^{N, R}\right)$ inherits the property of boundedness, independently on the parameter $R$, that holds for the limit object. If so, we can remove the cut-off, choosing $R$ large enough from the beginning. The first difficulty in the realization of this intuition is given by the type of convergence which we are dealing with: convergence in law. We will overcome this technicality, appealing to the Skorohod's Theorem to strengthen the convergence.

We will first state and prove a general result for almost sure convergence of random variables. Then, in order to utilize such criterion, we will make us Skorohod's Theorem and we will understand our particle systems in a path-by-path sense: we will give a precise definition of path-by-path solutions and prove a uniqueness result for such kind of solutions. The application of the above mentioned criterion to our case will let us transfer the property of convergence from the sequence $\left(u^{N, R}, S^{N, R}\right)$ to $\left(u^{N}, S^{N}\right)$.

In the rest of the section we will always assume to have taken

$$
R=\max \left(\mathbf{K}_{u}+1,\|u\|_{\infty}+1\right)
$$

where the constant $\mathbf{K}_{\mathbf{u}}$ has been defined in Proposition 5.13. This choice will assure that Proposition 5.1 is verified. The condition that $R$ is greater than $\|u\|_{\infty}+1$ is needed in order to let the sequence of $u^{N, R}$ to inherit the uniform boundedness of the limit $u$. This process will be clarified later.

\subsection{Convergence Criterion}

We now present the general criterion that we will use to obtain the convergence of the sequence $\left(u^{N}, S^{N}\right)_{N \in \mathbb{N}}$ from that of $\left(u^{N, R}, S^{N, R}\right)_{N \in \mathbb{N}}$. The framework of this criterion is pretty general. We preferred to isolate it an state it in its general form, rather than in our specific case, in order to make the underlying idea more evident. 
Theorem 6.1. (General Principle) Let $(\Omega, \mathcal{F}, \mathbf{P})$ a probability space and let $\left(E, d_{E}\right)$ a separable metric space. Let $\left\{X_{N}\right\}_{N \in \mathbb{N}}$ and $\left\{Y_{N}\right\}_{N \in \mathbb{N}}$ two sequences of random variables taking values in $E$ and let $x$ be a point in $E$. Moreover, suppose that for each $N \in \mathbb{N}$, there exist two collections of subset $S_{N}^{X}(\omega) \subseteq E$ and $S_{N}^{Y}(\omega) \subseteq E$, indexed by $\omega \in \Omega$. Assume further that the following conditions are satisfied:

1 .

$$
X_{N} \stackrel{N \rightarrow \infty}{\longrightarrow} x \in E \quad \mathbf{P} \text {-a.s. }
$$

2. denoting

$$
\Omega_{S}=\left\{\omega \in \Omega \mid \sharp S_{N}^{Y}(\omega) \leq 1 \quad \forall N \in \mathbb{N}\right\}
$$

where by $\sharp A$ we mean the cardinality of the set $A$, we have

$$
\mathbf{P}\left(\Omega_{S}\right)=1 ;
$$

3. denoting

$$
\begin{aligned}
& \Omega_{X}=\left\{\omega \in \Omega \mid X_{N}(\omega) \in S_{N}^{X}(\omega) \forall N \in \mathbb{N}\right\}, \\
& \Omega_{Y}=\left\{\omega \in \Omega \mid Y_{N}(\omega) \in S_{N}^{Y}(\omega) \forall N \in \mathbb{N}\right\},
\end{aligned}
$$

we have

$$
\mathbf{P}\left(\Omega_{X}\right)=\mathbf{P}\left(\Omega_{Y}\right)=1
$$

4.

$$
B_{E}(x, 1) \cap S_{N}^{X}(\omega) \subseteq S_{N}^{Y}(\omega) \quad \forall N \in \mathbb{N}, \forall \omega \in \Omega .
$$

Then the sequence $\left\{Y_{N}\right\}_{N \in \mathbb{N}}$ converges in $E$ to the same limit of the sequence $\left\{X_{N}\right\}_{N \in \mathbb{N}}$

$$
Y_{N} \stackrel{N \rightarrow \infty}{\longrightarrow} x \in E \quad \mathbf{P} \text {-a.s. }
$$

Proof. Consider the set

$$
\Omega_{C, X}:=\left\{\omega \in \Omega \mid d\left(X^{N}(\omega), x\right)_{E} \stackrel{N}{\rightarrow} 0\right\}
$$

and

$$
\Omega_{C, Y}:=\left\{\omega \in \Omega \mid d\left(Y^{N}(\omega), x\right)_{E} \stackrel{N}{\rightarrow} 0\right\}
$$

Note that, by property 1 . the set $\Omega_{C, X}$ has full measure $\mathbf{P}\left(\Omega_{C, X}\right)=1$.

We will prove that

$$
\Omega_{S} \cap \Omega_{C, X} \cap \Omega_{X} \cap \Omega_{Y} \subseteq \Omega_{C, Y}
$$

thus implying the thesis being $\mathbf{P}\left(\Omega_{S}\right)=\mathbf{P}\left(\Omega_{X}\right)=\mathbf{P}\left(\Omega_{Y}\right)=1$ by property 2 . and 3 . To do so let us consider the set

$$
\Omega_{1}=\left\{\omega \in \Omega \mid \exists N(\omega) d\left(X_{N}(\omega), x\right) \leq 1 \forall N>N(\omega)\right\}
$$

and note that

$$
\Omega_{X, C} \subseteq \Omega_{1}
$$

Now define

$$
\Omega_{2}=\left\{\omega \in \Omega \mid X_{N}(\omega)=Y_{N}(\omega) \forall N>N(\omega)\right\}
$$

where $N(\omega)$ is defined for each $\omega$, in the set $\Omega_{1}$. We claim that

$$
\Omega_{S} \cap \Omega_{X, C} \cap \Omega_{X} \cap \Omega_{Y} \subseteq \Omega_{2} .
$$

Take $\omega \in \Omega_{S} \cap \Omega_{X, C} \cap \Omega_{X} \cap \Omega_{Y}$. Hence if $N>N(\omega)$, given that $\omega$ lies in $\Omega_{X, C}$, it also lies in $\Omega_{1}$, thus we have $X_{N}(\omega) \in B_{E}(x, 1)_{E}$. Moreover, $\omega$ lies also in $\Omega_{X}$, hence $X_{N}(\omega) \in S_{N}^{X}(\omega)$. By property 4 . we conclude $X_{N}(\omega) \in S_{N}^{Y}(\omega)$. Furthermore $\omega \in \Omega_{Y}$ implies $Y_{N}(\omega) \in S_{N}^{Y}(\omega)$, but $\omega$ is also in $\Omega_{S}$ hence by property 2. $S_{N}^{Y}(\omega)$ is a singleton, hence $S_{N}^{Y}(\omega)=\left\{Y_{N}(\omega)\right\}$. Since $X_{N}(\omega) \in S_{N}^{X}(\omega)$ and $S_{N}^{Y}(\omega)=\left\{Y_{N}(\omega)\right\}$ we obtain $X_{N}(\omega)=Y_{N}(\omega)$ and we have proven condition (23). 
Finally, we can prove condition (22): taking $\omega \in \Omega_{S} \cap \Omega_{X, C} \cap \Omega_{X} \cap \Omega_{Y}$, we have that $\forall \varepsilon>0$ there exists $N_{\varepsilon}(\omega)$, such that

$$
d\left(X_{N}(\omega), x\right)_{E}<\varepsilon \quad \forall N>N_{\varepsilon}(\omega)
$$

By condition (23) $\omega$ lies also in $\Omega_{2}$, hence

$$
X_{N}(\omega)=Y_{N}(\omega) \quad \forall N>N(\omega) .
$$

Calling $\bar{N}(\omega)=\max \left(N_{\varepsilon}(\omega), N(\omega)\right)$ we conclude

$$
d\left(Y_{N}(\omega), x\right)_{E}<\varepsilon \quad \forall N>\bar{N}_{\varepsilon}(\omega)
$$

and hence $\omega \in \Omega_{Y, C}$. Thus the proof is concluded.

\subsection{Path by Path Solutions for $(P S-N S)$}

We will now focus on the problem of uniqueness for path-by-path solutions. The issue of uniqueness for this class of solutions is very difficult: very few result are know before the work of [7]. The analysis of such kind of problem for $(P S-N S)$ will be a key point of the proof of Theorem 2.3. In fact, to apply Theorem 6.1 to our case, we will see that strong uniqueness in the sense of SDEs, which is more classical than that path-by-path, will not be enough. We now recall the concept of path-by-path solutions and uniqueness in this class. We will discuss this topic in the specific case that is needed here, the system of PDE-SDEs $(P S-N S)$.

Recall system of equation $(P S-N S)$ and note that, in the equation for the particle position and velocity $\left(X_{t}^{i, N}, V_{t}^{i, N}\right)$ the noise is pure additive Brownian motion, i.e. the diffusion coefficient is constant. For this reason Itô integral is not involved into the equations and one can understand system of equations $(P S-N S)$ in its integral form as a coupling PDE-ODEs, where the Brownian motions plays the role of a given external force. This perspective is outlined in the following system

$$
\left\{\begin{array}{l}
\partial_{t} u^{N}=\Delta u^{N}-u^{N} \cdot \nabla u^{N}-\nabla \pi^{N}-\frac{1}{N} \sum_{i=1}^{N}\left(u_{\varepsilon_{N}}^{N}\left(X_{t}^{i, N}\right)-V_{t}^{i, N}\right) \delta_{X_{t}^{i, N}}^{\varepsilon_{N}} \\
\operatorname{div}\left(u^{N}\right)=0 \\
\left\{\begin{array}{l}
X_{t}^{i, N}=X_{0}^{i}+\int_{0}^{t} V_{s}^{i, N} d s \\
V_{t}^{i, N}=V_{0}^{i}+\int_{0}^{t}\left(u_{\varepsilon_{N}}^{N}\left(X_{s}^{i, N}\right)-V_{s}^{i, N}\right) d s+\sigma B_{t}^{i}(\omega)
\end{array}\right.
\end{array}\right.
$$

where $B_{t}^{i}(\omega)$ stands for a single realization of a Brownian path for fixed $\omega \in \Omega$. We now introduce the set of path-by-path solutions for a given realization of $\omega \in \Omega$ and for fixed $N \in \mathbb{N}$ :

$$
\begin{aligned}
& S_{N}(\omega)=\left\{\left(w,\left(x_{.}^{i}, v_{.}^{i}\right)_{i=1, \ldots, N}\right) \in C\left([0, T] \times \mathbb{T}^{2}\right) \times C\left([0, T] ; \mathbb{T}^{2} \times \mathbb{R}^{2}\right)^{N}\right. \text { s.t. } \\
& \left.\left(w,\left(x^{i}, v_{.}^{i}\right)_{i=1, \ldots, N}\right) \text { solves }(24) \text { with additive noise }\left(B_{t}^{i}(\omega)\right)_{i=1, \ldots, N}\right\} .
\end{aligned}
$$

Roughly speaking $S_{N}(\omega)$ is the set of curves that solves (24) in a deterministic setting for a prescribed realization of a Brownian path (identified by $\omega$ ). We do not give a precise definition of existence of pathby-path solutions. We remark that existence of weak or strong solutions in an SDE settings imply that the set $S_{N}(\omega)$ is non empty with probability one. We now focus our attention to the topic of uniqueness.

Definition 6.2. (Uniqueness of path-by-path solutions) Given a natural number $N$ we say that there is path-by-path uniqueness for system of equations $(P S-N S)$ with $N$ particles, if there exist a set $\Omega_{S} \subseteq \Omega$ with probability one $\mathbf{P}\left(\Omega_{S}\right)=1$ such that

$$
\sharp S_{N}(\omega) \leq 1 \quad \forall \omega \in \Omega_{S}
$$

where $\sharp A$ stands for the cardinality of the set $A$. 
Opposite to the case of existence, uniqueness of path-by-path solutions is a much more difficult topic: uniqueness in this class is a stronger notion that weak or strong uniqueness for SDE. In Definition 6.2 no measurability with respect to the probability space $\left(\Omega, \mathcal{F},\left\{\mathcal{F}_{t}\right\}, \mathbf{P}\right)$ is required. In case of uniqueness for SDE a much more richer structure is available, given that solutions are required at least to be adapted to the filtration $\mathcal{F}_{t}$.

We now prove a path-by-path uniqueness result for system of equation $(P S-N S)$. Some result about path-by-path uniqueness for SDEs are already known: Davie in [7] prove the result for a single SDE with pure additive Brownian noise and only bounded measurable drift. This type of result for low regularity drift functions, less than locally Lipschitz, are very difficult. In our case, the drift appearing into the particle equations $\left(X_{t}^{i, N}, V_{t}^{i, N}\right)$ is even more regular than Lipschitz: in fact the function $u_{\varepsilon_{N}}^{N}(t, x)$ is $C^{\infty}$ in the space variable due to the convolution with the $C^{\infty}$ function $\theta^{\varepsilon_{N}}(x)$. However, the case here is slightly different from the case of a single SDE due to the strong coupling with the Navier-Stokes equation that introduce additional difficulty.

Proposition 6.3. Let us consider on the probability space $\left(\Omega, \mathcal{F},\left\{\mathcal{F}_{t}\right\}, \mathbf{P}\right)$

$$
\Omega_{\mathcal{B}}=\left\{\omega \in \Omega \mid B_{t}^{i}(\omega) \text { is continuous on }[0, T] \forall i \in \mathbb{N}\right\} \subseteq \Omega
$$

the set where all the Brownian motion $\left(B^{i}\right)_{i \in \mathbb{N}}$ are continuous, which is of full measure with respect to $\mathbf{P}$. Then, for all $N \in \mathbb{N}$ we have uniqueness path-by-path for system of equation $(P S-N S)$ with $N$ particles, namely

$$
\sharp S_{N}(\omega) \leq 1 \quad \forall \omega \in \Omega_{\mathcal{B}} .
$$

Proof. For a matter of simplicity we prove the result in the case $N=1$ : the generalization for general $N$, is straightforward. Moreover, to make the notation less heavy, we will omit the dependence on $N$ and $\omega$ indicating with $u_{t}$ the variable $u_{t}^{N}(\omega)$ and with $\left(X_{t}, V_{t}\right)$ the couple of variables $\left(X_{t}^{1,1}, V_{t}^{1,1}\right)(\omega)$. Also the mollifier $\theta^{0, \varepsilon_{N}}$ will be labeled simply by $\theta$. In our simplification, the system becomes:

$$
\left\{\begin{array}{l}
\partial_{t} u=\Delta u-u \cdot \nabla u-\nabla \pi-\left((\theta * u)\left(X_{t}\right)-V_{t}\right) \theta\left(x-X_{t}\right) \\
\operatorname{div}(u)=0 \\
\left\{\begin{array}{l}
\dot{X}_{t}=V_{t} \\
\dot{V}_{t}=\left(\left(\theta * u_{t}\right)\left(X_{t}\right)-V_{t}\right)+B_{t} .
\end{array}\right.
\end{array}\right.
$$

Now we consider two solutions $(u, X, V)$ and $\left(u^{\prime}, X^{\prime}, V^{\prime}\right)$, with $\left(u_{0}, X_{0}, V_{0}\right)=\left(u_{0}^{\prime}, X_{0}^{\prime}, V_{0}^{\prime}\right)$, and we apply Gronwall Lemma to the quantity

$$
\left|X_{t}-X_{t}^{\prime}\right|+\left|V_{t}-V_{t}^{\prime}\right|+|| u_{t}-\left.u_{t}^{\prime}\right|_{H^{1+2 \alpha}\left(\mathbb{T}^{2}\right)},
$$

for $\alpha<\frac{1}{2}$.

We start by computing the distance of velocities, recalling that $V_{0}=V_{0}^{\prime}$ and $B_{t}$ is the same given function for the two solutions

$$
\begin{aligned}
\left|V_{t}-V_{t}^{\prime}\right| \leq & \int_{0}^{t}\left[\left(\theta * u_{s}\right)\left(X_{s}\right)-\left(\theta * u_{s}^{\prime}\right)\left(X_{s}^{\prime}\right)\right] d s+\int_{0}^{t}\left|V_{s}-V_{s}^{\prime}\right| d s \\
\leq & \int_{0}^{t}\left[\left(\theta * u_{s}\right)\left(X_{s}\right)-\left(\theta * u_{s}^{\prime}\right)\left(X_{s}\right)\right] d s+\int_{0}^{t}\left[\left(\theta * u_{s}^{\prime}\right)\left(X_{s}\right)-\left(\theta * u_{s}^{\prime}\right)\left(X_{s}^{\prime}\right)\right] d s \\
& +\int_{0}^{t}\left|V_{s}-V_{s}^{\prime}\right| d s \\
\lesssim & \int_{0}^{t}\left\|u_{s}-u_{s}^{\prime}\right\|_{H^{1+2 \alpha}\left(\mathbb{T}^{2}\right)} d s+\int_{0}^{t}\left|X_{s}-X_{s}^{\prime}\right| d s+\int_{0}^{t}\left|V_{s}-V_{s}^{\prime}\right| d s
\end{aligned}
$$

where we have used both the Lipschitzianity and boundedness in $L^{\infty}\left(\mathbb{T}^{2}\right)$ of $\theta * u_{s}$, as well as the embedding $H^{1+2 \alpha}\left(\mathbb{T}^{2}\right) \hookrightarrow C\left(\mathbb{T}^{2}\right)$. 
Regarding the $X$ component we simply have

$$
\left|X_{t}-X_{t}^{\prime}\right| \leq \int_{0}^{t}\left|V_{s}-V_{s}^{\prime}\right| d s .
$$

The main difficulty consists in estimating $\left\|u_{t}-u_{t}^{\prime}\right\|_{H^{1+2 \alpha}\left(\mathbb{T}^{2}\right)}$. As done in previous sections we approach the problem through the vorticity formulation. Call $\omega$ and $\omega^{\prime}$ the vorticity associated to $u$ and $u^{\prime}$. As in Lemma 5.9 , by the mild formulation of $\omega-\omega^{\prime}$ we have

$$
\begin{aligned}
\left\|\omega_{t}-\omega_{t}^{\prime}\right\|_{H^{2 \alpha}\left(\mathbb{T}^{2}\right)} \leq & \int_{0}^{t}\left\|(I-\Delta)^{\alpha} e^{(t-s) \Delta} u_{s} \cdot \nabla\left(\omega_{s}-\omega_{s}^{\prime}\right)\right\|_{L^{2}\left(\mathbb{T}^{2}\right)} d s \\
& +\int_{0}^{t}\left\|(I-\Delta)^{\alpha} e^{(t-s) \Delta}\left(u_{s}-u_{s}^{\prime}\right) \cdot \nabla \omega_{s}^{\prime}\right\|_{L^{2}\left(\mathbb{T}^{2}\right)} d s \\
& +\int_{0}^{t}\left\|(I-\Delta)^{\alpha} e^{(t-s) \Delta} \nabla^{\perp} \cdot \Lambda_{u, X, V}(s)\right\|_{L^{2}\left(\mathbb{T}^{2}\right)} d s
\end{aligned}
$$

where

$$
\Lambda_{u, X, V}(s):=\left[\left((\theta * u)\left(X_{s}\right)-V_{s}\right) \theta\left(x-X_{s}\right)-\left(\left(\theta * u^{\prime}\right)\left(X_{s}^{\prime}\right)-V_{s}^{\prime}\right) \theta\left(x-X_{s}^{\prime}\right)\right] .
$$

We now deal with each of the terms above separately. We strictly follow the same computation of Lemma 5.9, starting from (26):

$$
\begin{aligned}
(26) & \lesssim \int_{0}^{t} \frac{\left\|u_{s}\right\|_{C\left(\mathbb{T}^{2}\right)}|| \omega_{s}-\omega_{s}^{\prime} \|_{L^{2}\left(\mathbb{T}^{2}\right)}}{|t-s|^{\alpha+1 / 2}} d s \\
& \lesssim\|u\|_{\infty} \int_{0}^{t} \frac{\left\|u_{s}-u_{s}^{\prime}\right\|_{H^{1+2 \alpha}\left(\mathbb{T}^{2}\right)}}{|t-s|^{\alpha+1 / 2}} d s \\
(27) & \lesssim \int_{0}^{t} \frac{\left\|u_{s}-u_{s}^{\prime}\right\|_{C\left(\mathbb{T}^{2}\right)}|| \omega_{s}^{\prime} \|_{L^{2}\left(\mathbb{T}^{2}\right)}}{|t-s|^{\alpha+1 / 2}} d s \\
& \lesssim\left\|\omega^{\prime}\right\|_{L^{\infty}\left([0, T] ; L^{2}\left(\mathbb{T}^{2}\right)\right)} \int_{0}^{t} \frac{\left\|u_{s}-u_{s}^{\prime}\right\|_{H^{1+2 \alpha}\left(\mathbb{T}^{2}\right)}}{|t-s|^{\alpha+1 / 2}} d s .
\end{aligned}
$$

In the same way we have

$$
(28) \lesssim \int_{0}^{t} \frac{\left\|\Lambda_{u, X, V}(s)\right\|_{L^{2}\left(\mathbb{T}^{2}\right)}}{|t-s|^{\alpha+1 / 2}} d s
$$

We proceed now by adding and subtracting the right quantities from $\Lambda_{u, X, V}(s)$ obtaining

$$
\begin{aligned}
& \left|\left[\left(\theta * u_{s}\right)\left(X_{s}\right)-V_{s}\right] \theta\left(x-X_{s}\right)-\left[\left(\theta * u_{s}^{\prime}\right)\left(X_{s}^{\prime}\right)-V_{s}^{\prime}\right] \theta\left(x-X_{s}^{\prime}\right)\right| \leq \\
& \quad \leq \theta\left(x-X_{s}\right)\left|\left(\theta * u_{s}\right)\left(X_{s}\right)-\left(\theta * u_{s}^{\prime}\right)\left(X_{s}\right)\right| \\
& \quad+\theta\left(x-X_{s}\right)\left|\left(\theta * u_{s}^{\prime}\right)\left(X_{s}\right)-\left(\theta * u_{s}^{\prime}\right)\left(X_{s}^{\prime}\right)\right| \\
& \quad+u_{s}^{\prime}\left(X_{s}^{\prime}\right)\left|\theta\left(x-X_{s}\right)-\theta\left(x-X_{s}^{\prime}\right)\right| \\
& \quad+\theta\left(x-X_{s}\right)\left|V_{s}-V_{s}^{\prime}\right| \\
& \quad+\left|V_{s}\right|\left|\theta\left(x-X_{s}\right)-\theta\left(x-X_{s}^{\prime}\right)\right| \\
& \quad \lesssim|| u_{s}-\left.u_{s}^{\prime}\right|_{H^{1+2 \alpha}\left(\mathbb{T}^{2}\right)}+\left|X_{s}-X_{s}^{\prime}\right|+\left|V_{s}-V_{s}^{\prime}\right|
\end{aligned}
$$

by using the boundedness of $u$ and $u^{\prime}$, the Lipschitzianity of $(\theta * u)$, the boundedness of $\left|V_{s}\right|$ and that of $\theta$. Hence we obtained

$$
(28) \lesssim \int_{0}^{t} \frac{|| u_{s}-\left.u_{s}^{\prime}\right|_{H^{1+2 \alpha}\left(\mathbb{T}^{2}\right)}+\left|X_{s}-X_{s}^{\prime}\right|+\left|V_{s}-V_{s}^{\prime}\right|}{|t-s|^{1 / 2+\alpha}} d s
$$


We conclude by a standard Gronwall type inequality.

\subsection{Proof of Theorem 2.3}

We finally have all the ingredients to prove Theorem 2.3. Since the proof is quite technical we first outline the general strategy.

From Proposition 5.1 we have obtained convergence in Law of the sequence $\left(u^{N, R}, S^{N, R}\right)$ to the unique weak solution of $(V N S)$, call it $(u, F)$. We aim to obtain the same result for the sequence $\left(u^{N}, S^{N}\right)$, namely to prove Theorem 2.3. To do so, we will apply the convergence criterion stated in Theorem 6.1 , to transfer the convergence from one sequence to another. However, Theorem 6.1 requires the sequences of random variables involved, to converge almost surely in the appropriate topology, while Proposition 5.1 grants us only convergence in law. Hence, to overcome this problem, we will first appeal to a slight variation of Skorohod representation Theorem, Lemma 6.4, applied to the sequence $\left(u^{N, R}, S^{N, R}\right)_{N \in \mathbb{N}}$ in order to obtain almost sure convergence from convergence in law. Let us omit some technicalities concerning Skorohod Theorem, whose details will be clarified later, and assume now that the sequence $\left(u^{N, R}, S^{N, R}\right)$ is converging almost surely to $(u, F)$. We will apply Theorem 6.1 by taking

$$
X_{N}=\left(u^{N, R}, S^{N, R}\right), \quad Y_{N}=\left(u^{N}, S^{N}\right), \quad x=(u, F) .
$$

Still avoiding some technicalities we will chose

$$
S_{N}^{X}(\omega)=\text { the set of path-by-path solutions of }\left(P S^{R}-N S^{R}\right)
$$

and

$$
S_{N}^{Y}(\omega)=\text { the set of path-by-path solutions of }(P S-N S) .
$$

With this choice we will see that conditions [1-4] stated in Theorem 6.1 will be satisfied. We can now outline the reasoning behind the hypotheses of Theorem 6.1 in the following scheme:

Condition 1. corresponds to Proposition 5.1, that is the convergence of $\left(u^{N, R}, S^{N, R}\right)$ to the limit point $(u, F)$;

Condition 2. resembles to the path-by-path uniqueness result, Proposition 6.3;

Condition 3. states that $\left(u^{N, R}, S^{N, R}\right)$ is a path-by-path solution of $\left(P S^{R}-N S^{R}\right)$ and the analogue for $\left(u^{N}, S^{N}\right)$

Condition 4. expresses the fact that path-by-path solutions of $\left(P S^{R}-N S^{R}\right)$ which are also bounded from above, also satisfies $(P S-N S)$ if the parameter $R$ is large enough. This is the same idea used to prove Theorem 5.14 when we proved that two PDE system coincide for large $R$.

We now remark the importance of dealing with path-by-path uniqueness. Imagine to replace condition 2. in Theorem 6.1, with some condition that mimics the idea of strong uniqueness for SDE, instead of that for path-by-path uniqueness. A possible modification is the following:

Condition 2.bis: For all $N \in \mathbb{N}$ and for every $Z E$-valued random variable, if

$$
\mathbf{P}\left(Z(\omega) \in S_{N}^{Y}(\omega)\right)=1
$$

then

$$
\mathbf{P}\left(Z(\omega)=Y_{N}(\omega)\right)=1 .
$$

Now, following the proof of Theorem 6.1, we can proceed into the proof up to a certain point. Specifically we can prove that the set

$$
\left\{X_{N}(\omega) \in S_{N}^{Y}(\omega) \quad \forall N>N(\omega)\right\}
$$

is of full measure with respect to $\mathbf{P}$. However, there is no way to apply condition 2.bis, to conclude that

$$
\mathbf{P}\left(X_{N}(\omega)=Y_{N}(\omega) \quad \forall N>N(\omega)\right)=1
$$

as it would be needed to end the proof. 
We now recall and prove a small variation of Skorohod's Theorem, that we will need in the proof of Theorem 2.3.

Lemma 6.4. Let $(\Omega, \mathcal{F}, \mathbf{P})$ be a probability space and let $\left(X_{N}, Y_{N}\right)_{N \in \mathbb{N}}$ be a sequence of random variables defined on $\Omega$, taking values on a separable metric space $E \times F$. Assume that $F$ is also a Banach space and $Y_{N} \in L^{1}(\Omega ; F)$ for each $N \in \mathbb{N}$. Let also $X: \Omega \rightarrow E$ be a random variable and assume further that

$$
X_{N} \stackrel{\mathcal{L} a w}{\rightarrow} X .
$$

Then, there exist a probability space $(\widetilde{\Omega}, \widetilde{\mathcal{F}}, \widetilde{\mathbf{P}})$ and random variables defined on the new probability space $\left(\widetilde{X}_{N}, \widetilde{Y}_{N}\right)_{N \in \mathbb{N}}, \widetilde{X}$ such that

$$
\left(\widetilde{X}_{N}, \widetilde{Y}_{N}\right) \stackrel{\mathcal{L} a w}{\sim}\left(X_{N}, Y_{N}\right), \quad \widetilde{X} \stackrel{\mathcal{L} a w}{\sim} X
$$

and

$$
\widetilde{X}_{N} \rightarrow \widetilde{X} \quad \widetilde{\mathbf{P}} \text {-almost-surely. }
$$

Proof. The proof relies on the classical Skorohod's Theorem, see [4].

Call $c_{N}:=\mathbf{E}\left[\left\|Y_{N}\right\|_{F}\right]$, and introduce $a_{N}=N c_{N}$. Consider now the sequence $Z_{N}:=Y_{N} / a_{N}$ and notice that

$$
Z_{N} \stackrel{\mathcal{L} a w}{\rightarrow} 0
$$

since the convergence also holds in probability. Now, applying Skorohod's Theorem to the sequence $\left(X_{N}, Z_{N}\right)$ we obtain that there exist a new probability space $(\widetilde{\Omega}, \widetilde{\mathcal{F}}, \widetilde{\mathbf{P}})$ and random variables $\left(\widetilde{X}_{N}, \widetilde{Z}_{N}\right)_{N \in \mathbb{N}}$, $\widetilde{X}$ such that

$$
\left(\widetilde{X}_{N}, \widetilde{Z}_{N}\right) \stackrel{\mathcal{L} a w}{\sim}\left(X_{N}, Z_{N}\right), \quad \widetilde{X} \stackrel{\mathcal{L} a w}{\sim} X
$$

and

$$
\widetilde{X}_{N} \rightarrow \widetilde{X} \quad \widetilde{\mathbf{P}} \text {-almost-surely. }
$$

Introduce $\widetilde{Y}_{N}:=a_{N} \widetilde{Z}_{N}$ and observe that $\left(\widetilde{X}_{N}, \widetilde{Y}_{N}\right) \stackrel{\mathcal{L} a w}{\sim}\left(X_{N}, Y_{N}\right)$. This concludes the proof.

Proof of Theorem 2.3. As explained the above the proof is divided into three steps: first we apply Lemma 6.4 to the sequence $\left(u^{N, R}, S^{N, R}\right)$ to obtain almost sure convergence on a new probability space. Second, we will see that the new random variables obtained, on the new probability space satisfies the same equations as the original one. Lastly, we apply the general principle Theorem 6.1 to transfer the convergence from $\left(u^{N, R}, S^{N, R}\right)$ to $\left(u^{N}, S^{N}\right)$.

Step 1: Let us first introduce some notation. For each $N \in \mathbb{N}$ we first introduce $\mathbf{X}^{N, R} \in C\left([0, T] ; \mathbb{T}^{2}\right)^{\mathbb{N}}$ and $\mathbf{V}^{N, R} \in C\left([0, T] ; \mathbb{R}^{2}\right)^{\mathbb{N}}$ defined as

$$
\mathbf{X}^{N, R}(i)=\left\{\begin{array}{ll}
X^{i, N, R} & \text { if } i \leq N, \\
0 & \text { otherwise, }
\end{array} \quad \mathbf{V}^{N, R}(i)= \begin{cases}V^{i, N, R} & \text { if } i \leq N, \\
0 & \text { otherwise }\end{cases}\right.
$$

where 0 stands for the function which is identically zero. Roughly speaking $\mathbf{X}^{N, R}$ (respectively $\mathbf{V}^{N, R}$ ) is the sequence of functions, where the first $N$ elements are the particles trajectories $X^{i, N, R}$, and all the others are identically zero. Now we apply Lemma 6.4 to the sequence

$$
\left(u^{N, R}, S^{N, R},\left(B^{i}\right)_{i \in \mathbb{N}}, \mathbf{X}^{N, R}, \mathbf{V}^{N, R}\right)_{N \in \mathbb{N}}
$$

where

$$
\left(u^{N, R}, S^{N, R},\left(B^{i}\right)_{i \in \mathbb{N}}\right) \stackrel{\mathcal{L} a w}{\longrightarrow}\left(u, F,\left(B^{i}\right)_{i \in \mathbb{N}}\right)
$$


and we just need to verify that $\left(\mathbf{X}^{N, R}, \mathbf{V}^{N, R}\right)$ is integrable with respect to $\mathbf{P}$ for each $N \in \mathbb{N}$. However this is true because

$$
\begin{aligned}
\mathbf{E}\left[\left\|\mathbf{X}^{N, R}\right\|_{L^{\infty}\left([0, T] ; \mathbb{T}^{2}\right)^{\mathbb{N}}}\right. & =\mathbf{E}\left[\max _{i \leq N} \sup _{t \in[0, T]}\left\|X_{t}^{i, N, R}\right\|\right] \\
& \leq N \mathbf{E}\left[\sup _{t \in[0, T]}\left\|X_{t}^{i, N, R}\right\|\right] \leq C \cdot N
\end{aligned}
$$

by using exchangeability and by the fact that $\mathbf{E}\left[\sup _{t \in[0, T]}\left\|X_{t}^{i, N, R}\right\|\right] \leq C$ due to the presence of the cutoff in system of equations $\left(P S^{R}-N S^{R}\right)$. The same result holds for every $\mathbf{V}^{N, R}$ by using the same argument.

We can now apply Lemma 6.4 . Hence there exists a new filtered probability space $\left(\widetilde{\Omega}, \widetilde{\mathcal{F}},\left\{\widetilde{\mathcal{F}}_{t}\right\}, \widetilde{\mathbf{P}}\right)$ and new sequences of random variables

$$
\left(\widetilde{u}^{N, R}, \widetilde{S}^{N, R},\left(\widetilde{B}^{i, N}\right)_{i \leq N}, \widetilde{\mathbf{X}}^{N, R}, \widetilde{\mathbf{V}}^{N, R}\right)_{N \in \mathbb{N}}
$$

that shares the same laws of the initial sequences

$$
\left(\widetilde{u}^{N, R}, \widetilde{S}^{N, R},\left(\widetilde{B}^{i, N}\right)_{i \leq N}, \widetilde{\mathbf{X}}^{N, R}, \widetilde{\mathbf{V}}^{N, R}\right) \stackrel{\mathcal{L} a w}{\sim}\left(u^{N, R}, S^{N, R},\left(B^{i}\right)_{i \leq N}, \mathbf{X}^{N, R}, \mathbf{V}^{N, R}\right)
$$

for all $N \in \mathbb{N}$, and that satisfies

$$
\left(\widetilde{u}^{N, R}, \widetilde{S}^{N, R}\right) \stackrel{N \rightarrow \infty}{\longrightarrow}(u, F) \quad \widetilde{\mathbf{P}} \text {-a.s. }
$$

Step 2: We now verify that the new random variables satisfies the same equations as the original ones, namely system of equations $\left(P S^{R}-N S^{R}\right)$. Moreover, in order to apply Theorem 6.1 we also need to have on the new probability space an analogue of $\left(u^{N}, S^{N}\right)$, that still satisfies system of equations $(P S-N S)$ and of which we will prove the convergence. Namely:

1. Denoting by $\widetilde{X}^{i, N, R}$ and $\widetilde{V}^{i, N, R}$ the first $N$ components of $\left(\widetilde{\mathbf{X}}^{N, R}, \widetilde{\mathbf{V}}^{N, R}\right)$, corresponding to those that are non zero, we need to check that, for every $N \in \mathbb{N}$

$$
\widetilde{S}_{t}^{N, R}=\frac{1}{N} \sum_{i=1}^{N} \delta_{\left(\widetilde{X}_{t}^{i, N, R}, \widetilde{V}_{t}^{i, N, R}\right)} .
$$

To prove this, consider the functional $\Phi^{S, N}$ defined as:

$$
\begin{aligned}
& \Phi^{S, N}\left(S^{N, R},\left(X^{i, N, R}\right)_{i \leq N},\left(V^{i, N, R}\right)_{i \leq N}\right) \\
& :=\sup _{\varphi \in C_{b}\left(\mathbb{T}^{2} \times \mathbb{R}^{2}\right)} \sup _{t \in[0, T]}\left|\left\langle S^{N, R}, \varphi\right\rangle-\frac{1}{N} \sum_{i=1}^{N} \varphi\left(X_{t}^{i, N, R}, V_{t}^{i, N, R}\right)\right|
\end{aligned}
$$

which is a measurable functional, and note that this is identically zero by definition of $S^{N, R}$. Now, by the fact that the random vectors $\left(S^{N, R},\left(X^{i, N, R}\right)_{i \leq N},\left(V^{i, N, R}\right)_{i \leq N}\right)$ and $\left(\widetilde{S}^{N, R},\left(\widetilde{X}^{i, N, R}\right)_{i \leq N}\right.$, $\left.\left(\widetilde{V}^{i, N, R}\right)_{i \leq N}\right)$ share the same law, we have

$$
\begin{aligned}
& \mathbb{E}^{\widetilde{\mathbf{P}}}\left[\Phi^{S, N}\left(\widetilde{S}^{N, R},\left(\widetilde{X}^{i, N, R}\right)_{i \leq N},\left(\widetilde{V}^{i, N, R}\right)_{i \leq N}\right)\right] \\
& \quad=\mathbb{E}^{\mathbf{P}}\left[\Phi^{S, N}\left(S^{N, R},\left(X^{i, N, R}\right)_{i \leq N},\left(V^{i, N, R}\right)_{i \leq N}\right)\right]=0 .
\end{aligned}
$$

Hence, we conclude that $\Phi^{S, N}\left(\widetilde{S}^{N, R},\left(\widetilde{X}^{i, N, R}\right)_{i \leq N},\left(\widetilde{V}^{i, N, R}\right)_{i \leq N}\right)$ is identically zero $\widetilde{\mathbf{P}}$-almost surely, which implies (29).

2. To prove that the new object satisfies the same equation as the initial one, for each $N \in \mathbb{N}$ we consider a bounded and measurable functional $\Phi^{N}$ taking as argument the function $u^{N, R}$, the particles $\left(X^{i, N, R}\right)_{i \leq N},\left(V^{i, N, R}\right)_{i \leq N}$ and the Brownian motions $\left(B^{i}\right)_{i \leq N}$, that vanishes in expected value on solutions of system of equation $\left(P S^{R}-N S^{R}\right)$. The measurability of $\Phi^{N}$ follows by the path-bypath formulation while the boundedness requirement can be dealt with by considering a sequence 
$\Phi^{M, N}:=\Phi^{N} \wedge M$ and passing to the limit in $M$ inside the expected value by monotone convergence. By the equality in law of the new sequences with respect to the initial one, we have that the functional $\Phi^{N}$ vanishes also on the new objects, when averaged with respect to $\widetilde{\mathbf{P}}$, namely (we omit some technical details of integrability)

$$
\begin{aligned}
\mathbb{E}^{\widetilde{\mathbf{P}}}\left[\Phi^{N}\left(\widetilde{u}^{N, R},\left(\widetilde{X}^{i, N, R}\right)_{i \leq N},\left(\widetilde{V}^{i, N, R}\right)_{i \leq N},\left(\widetilde{B}^{i, N}\right)_{i \leq N}\right)\right] \\
\left.\quad=\mathbb{E}^{\mathbf{P}}\left[\Phi^{N}\left(u^{N, R},\left(X^{i, N, R}\right)_{i \leq N},\left(V^{i, N, R}\right)_{i \leq N}\right),\left(B^{i}\right)_{i \leq N}\right)\right]=0 .
\end{aligned}
$$

Hence, $\left.\left(\widetilde{u}^{N, R},\left(\widetilde{X}^{i, N, R}\right)_{i \leq N},\left(\widetilde{V}^{i, N, R}\right)_{i \leq N}\right),\left(\widetilde{B}^{i, N}\right)_{i \leq N}\right)$ satisfies system of equation $\left(P S^{R}-N S^{R}\right)$ in the new probability space $\left(\widetilde{\Omega}, \widetilde{\mathcal{F}},\left\{\widetilde{\mathcal{F}}_{t}\right\}, \widetilde{\mathbf{P}}\right)$ which ends this part.

3. Consider now the sequence $\left.\left(u^{N},\left(X^{i, N}\right)_{i \leq N},\left(V^{i, N}\right)_{i \leq N}\right)\right)_{N \in \mathbb{N}}$, associated with system of equations $(P S-N S)$, that is the particle system without the cut-off. On the new probability space $\left(\widetilde{\Omega}, \widetilde{\mathcal{F}},\left\{\widetilde{\mathcal{F}}_{t}\right\}, \widetilde{\mathbf{P}}\right)$ consider the same system of equations $(P S-N S)$, i.e. the system of equation where the Brownian motions $\left(B^{i}\right)_{i \leq N}$ are replaced by the Brownian motions $\left(\widetilde{B}^{i, N}\right)_{i \leq N}$ introduced in Step 1. Call $\left.\left(\widetilde{u}^{N},\left(\widetilde{X}^{i, N}\right)_{i \leq N},\left(\tilde{\widetilde{V}}^{i, N}\right)_{i \leq N}\right)\right)_{N \in \mathbb{N}}$ the solution of such system, which can be seen as a random variable on $\left(\widetilde{\Omega}, \widetilde{\mathcal{F}},\left\{\widetilde{\mathcal{F}}_{t}\right\}, \widetilde{\mathbf{P}}\right)$. Since solutions of system $(P S-N S)$ are unique in law we conclude that for all $N \in \mathbb{N}$

$$
\left.\left.\left(\widetilde{u}^{N},\left(\widetilde{X}^{i, N}\right)_{i \leq N},\left(\widetilde{V}^{i, N}\right)_{i \leq N}\right)\right) \stackrel{\mathcal{L} a w}{\sim}\left(u^{N},\left(X^{i, N}\right)_{i \leq N},\left(V^{i, N}\right)_{i \leq N}\right)\right) .
$$

Also introduce the analogue of the empirical measure $S^{N}$ on the new space

$$
\widetilde{S}_{t}^{N}:=\frac{1}{N} \sum_{i=1}^{N} \delta_{\left(\widetilde{X}_{t}^{i, N}, \widetilde{V}_{t}^{i, N}\right)} .
$$

By the previous definition and by construction of $\left(\left(X^{i, N}\right)_{i \leq N},\left(V^{i, N}\right)_{i \leq N}\right)$ we immediately have

$$
\left(u^{N}, S^{N}\right) \stackrel{\mathcal{L} a w}{\sim}\left(\widetilde{u}^{N}, \widetilde{S}^{N}\right), \quad \forall N \in \mathbb{N} .
$$

Step 3: We can now apply Theorem 6.1. We have to define all the objects needed in the Theorem and verify all the four conditions required. Let $E=C\left([0, T] \times \mathbb{T}^{2}\right) \times C\left([0, T] ; \mathbf{P}_{1}\left(\mathbb{T}^{2} \times \mathbb{R}^{2}\right)\right.$ and let $x \in E$ be the couple $(u, F)$. Now we take

$$
X_{N}:=\left(\widetilde{u}^{N, R}, \widetilde{S}^{N, R}\right), \quad Y_{N}:=\left(\widetilde{u}^{N}, \widetilde{S}^{N}\right) .
$$

Now define, for $\widetilde{\omega} \in \widetilde{\Omega}$

$$
\begin{aligned}
& S_{N}^{R}(\widetilde{\omega})=\left\{\left(w,\left(x^{i}\right)_{i \leq N},\left(v^{i}\right)_{i \leq N}\right) \in C\left([0, T] \times \mathbb{T}^{2}\right) \times C\left([0, T] ; \mathbb{T}^{2} \times \mathbb{R}^{2}\right)^{N}\right. \text { s.t. } \\
& \left.\left(w,\left(x^{i}\right)_{i \leq N},\left(v^{i}\right)_{i \leq N}\right) \text { solves }\left(P S^{R}-N S^{R}\right) \text { with additive noise }\left(B_{t}^{i}(\widetilde{\omega})\right)_{i \leq N}\right\},
\end{aligned}
$$

the set of path-by-path solutions for system of equations $\left(P S^{R}-N S^{R}\right)$. We also introduce the analogue for $(P S-N S)$ : call it $S_{N}(\widetilde{\omega})$. Now we consider

$$
S_{N}^{X}(\widetilde{\omega}):=\left\{(w, \mu) \in E \mid \mu=\frac{1}{N} \sum_{i=1}^{N} \delta_{\left(x^{i}, v^{i}\right)},\left(w,\left(x^{i}\right)_{i \leq N},\left(v^{i}\right)_{i \leq N}\right) \in S_{N}^{R}(\widetilde{\omega})\right\},
$$

and

$$
S_{N}^{Y}(\widetilde{\omega}):=\left\{(w, \mu) \in E \mid \mu=\frac{1}{N} \sum_{i=1}^{N} \delta_{\left(x^{i}, v^{i}\right)},\left(w,\left(x^{i}\right)_{i \leq N},\left(v^{i}\right)_{i \leq N}\right) \in S_{N}(\widetilde{\omega})\right\} .
$$

Roughly speaking, $S_{N}^{X}\left(\operatorname{resp} . S_{N}^{Y}\right)$ are the set of couples $(w, \mu)$ where $u$ is a function and $\mu$ is a measure, such that $\mu$ is the empirical measure of a set of particles which, together with $u$, are path-by-path solutions 
of $\left(P S^{R}-N S^{R}\right)$. This is just a way of rewriting sets of path-by-path solutions, which match the structure of the space $E$ where the converging objects belong.

Now we just need to verify rigorously all the four conditions stated in this Theorem:

1. In the first Step of this proof, we saw that

$$
\left(\widetilde{u}^{N, R}, \widetilde{S}^{N, R}\right) \stackrel{N \rightarrow \infty}{\longrightarrow}(u, F) \quad \widetilde{\mathbf{P}} \text {-a.s. }
$$

which correspond exactly to condition 1.

2. Introduce

$$
\Omega_{B}=\left\{\widetilde{\omega} \in \widetilde{\Omega} \mid\left(\widetilde{B}^{i, N}(\widetilde{\omega})\right)_{i \leq N, N \in \mathbb{N}} \text { are continuous }\right\}
$$

and note that, since we are considering a countable set of Brownian Motions, this set is of full measure with respect to $\widetilde{\mathbf{P}}$. Then, by Proposition 6.3 , we have that

$$
\sharp S_{N}(\widetilde{\omega}) \leq 1 \quad \forall \widetilde{\omega} \in \Omega_{B} .
$$

Hence, the same result holds for the set $S_{N}^{Y}(\widetilde{\omega})$.

3. Condition 3. states that $\left(\widetilde{u}^{N, R}, \widetilde{S}^{N, R}\right)$ belongs to the set $S_{N}^{X}$ almost surely. However, in Step 2. of this proof we have verified that on $\left(\widetilde{\Omega}, \widetilde{\mathcal{F}},\left\{\widetilde{\mathcal{F}}_{t}\right\}, \widetilde{\mathbf{P}}\right)$

$\left.\left(\widetilde{u}^{N, R},\left(\widetilde{X}^{i, N, R}\right)_{i \leq N},\left(\widetilde{V}^{i, N, R}\right)_{i \leq N}\right),\left(\widetilde{B}^{i, N}\right)_{i \leq N}\right)$ satisfies system of equation $\left(P S^{R}-N S^{R}\right)$ in the sense of SDEs. This condition implies that for fixed $\widetilde{\omega} \in \widetilde{\Omega}$ the vector $\left(\widetilde{u}^{N, R}(\widetilde{\omega})\right.$, $\left.\left(\widetilde{X}^{i, N, R}(\widetilde{\omega})\right)_{i \leq N},\left(\widetilde{V}^{i, N, R}(\widetilde{\omega})\right)_{i \leq N}\right) \in S_{N}^{R}(\widetilde{\omega})$. Since in Step 2. we verified that $\widetilde{S}^{N, R}$ is in fact an empirical measures on particle solutions of $\left(P S^{R}-N S^{R}\right)$ and by the definition of $S_{N}^{X}$, this imply the first part of condition 3. The same result holds for $\left(\widetilde{u}^{N}, \widetilde{S}^{N}\right)$ and $S_{N}^{Y}$ by an analogous argument.

4. Condition 4. is the most delicate. Take a couple $(w, \mu) \in S_{N}^{X}(\widetilde{\omega}) \cap B_{E}((u, F), 1)$. Since $(w, \mu) \in$ $B_{E}((u, F), 1)$ we have that

$$
\|w\|_{C\left([0, T] \times \mathbb{T}^{2}\right)} \leq\|u\|_{C\left([0, T] \times \mathbb{T}^{2}\right)}+1
$$

The couple $(w, \mu)$ also lies in $S_{N}^{X}(\widetilde{\omega})$, hence there exist $\left(\left(x^{i}\right),\left(v^{i}\right)\right)_{i \leq N} \in C\left([0, T] ; \mathbb{T}^{2} \times \mathbb{R}^{2}\right)$ such that $\left(w,\left(x^{i}\right)_{i \leq N},\left(v^{i}\right)_{i \leq N}\right) \in S_{N}^{R}(\widetilde{\omega})$, which means that is a path-by-bath solutions of $\left(P S^{R}-N S^{R}\right)$. However, since $w$ is uniformly bounded by $\|u\|_{C\left([0, T] \times \mathbb{T}^{2}\right)}+1$, which corresponds exactly to our choice of $R$ (see at the beginning of this section), we see that the cut off function $\chi_{R}(w) \equiv 1$ is identically one. Hence system of equation $\left(P S^{R}-N S^{R}\right)$ reduce to $(P S-N S)$, which is the particle system without the cut-off. This implies that $\left(w,\left(x^{i}\right)_{i \leq N},\left(v^{i}\right)_{i \leq N}\right)$ solves also $(P S-N S)$, hence $(w, \mu) \in S_{N}^{Y}(\widetilde{\omega})$.

Since we verified all the necessary conditions, we can apply Theorem 6.1, obtaining

$$
\left(\widetilde{u}^{N}, \widetilde{S}^{N}\right) \stackrel{N \rightarrow \infty}{\longrightarrow}(u, F) \quad \widetilde{\mathbf{P}} \text {-a.s. }
$$

Since almost sure convergence implies convergence in law, and since we verified in Step 2. that

$$
\left(u^{N}, S^{N}\right) \stackrel{\mathcal{L} a w}{\sim}\left(\widetilde{u}^{N}, \widetilde{S}^{N}\right), \quad \forall N \in \mathbb{N} .
$$

we can transport this type of convergence to the original probability space $\left(\Omega, \mathcal{F},\left\{\mathcal{F}_{t}\right\}, \mathbf{P}\right)$, hence the proof is ended. 
Funding Open access funding provided by Scuola Normale Superiore within the CRUI-CARE Agreement.

\section{Compliance with Ethical Standards}

Conflict of interest The authors declare that there is no conflict of interest.

Open Access. This article is licensed under a Creative Commons Attribution 4.0 International License, which permits use, sharing, adaptation, distribution and reproduction in any medium or format, as long as you give appropriate credit to the original author(s) and the source, provide a link to the Creative Commons licence, and indicate if changes were made. The images or other third party material in this article are included in the article's Creative Commons licence, unless indicated otherwise in a credit line to the material. If material is not included in the article's Creative Commons licence and your intended use is not permitted by statutory regulation or exceeds the permitted use, you will need to obtain permission directly from the copyright holder. To view a copy of this licence, visit http://creativecommons.org/licenses/by/4.0/.

Publisher's Note Springer Nature remains neutral with regard to jurisdictional claims in published maps and institutional affiliations.

\section{References}

[1] Allaire, G.: Homogenization of the Navier-Stokes equations in open sets perforated with tiny holes I. Abstract framework, a volume distribution of holes. Arch. Ration. Mech. Anal. 113(3), 209-259 (1991)

[2] Allaire, G.: Homogenization of the Navier-Stokes equations in open sets perforated with tiny holes II: non-critical sizes of the holes for a volume distribution and a surface distribution of holes. Arch. Ration. Mech. Anal. 113(3), 261-298 (1991)

[3] Bernard, E., Desvillettes, L., Golse, F., Ricci, V.: A derivation of the Vlasov-Navier-Stokes model for aerosol flows from kinetic theory. Communications in Mathematical Sciences 15(6), 1703-1741 (2017)

[4] Billingsley, P.: Convergence of Probability Measures. Wiley, Hoboken (2013)

[5] Boudin, L., Desvillettes, L., Grandmont, C., Moussa, A.: Global existence of solutions for the coupled Vlasov and Navier-Stokes equations. Differ. Integr. Equ. 22(11/12), 1247-1271 (2009)

[6] Chae, M., Kang, K., Lee, J.: Global existence of weak and classical solutions for the Navier-Stokes-Vlasov-FokkerPlanck equations. J. Differ. Equ. 251(9), 2431-2465 (2011)

[7] Davie, A.M.: Uniqueness of solutions of stochastic differential equations. International Mathematics Research Notices (2007)

[8] Degond, P.: Global existence of smooth solutions for the Vlasov-Fokker-Planck equation in 1 and 2 space dimensions. Annales scientifiques de l'École Normale Supérieure 19, 519-542 (1986)

[9] Desvillettes, L., Golse, F., Ricci, V.: The mean-field limit for solid particles in a Navier-Stokes flow. J. Stat. Phys. 131(5), 941-967 (2008)

[10] Desvillettes, L., Mathiaud, J.: Some aspects of the asymptotics leading from gas-particles equations towards multiphase flows equations. J. Stat. Phys. 141(1), 120-141 (2010)

[11] Feireisl, E., Namlyeyeva, Y., Nečasová, Š.: Homogenization of the evolutionary Navier-Stokes system. Manuscr. Math. 149(1-2), 251-274 (2016)

[12] Flandoli, F.: Random perturbation of pdes and fluid dynamic models. In: Ecole d'été de probabilités de Saint-Flour 2010, vol. 2015. Springer (2011)

[13] Flandoli, F.: A fluid-particle system related to Vlasov-Navier-Stokes equations. In: Maekawa, Y. (ed.) Mathematical Analysis of Viscous Incompressible Fluid, RIMS Kôkyûroku 2058. RIMS, Kyoto (2017)

[14] Flandoli, F., Leocata, M., Ricci, C.: The Vlasov-Navier-Stokes equations as a mean field limit. In: Discrete and Continuous Dynamical Systems - B, 22 (2017)

[15] Flandoli, F., Mahalov, A.: Stochastic three-dimensional rotating Navier-Stokes equations: averaging, convergence and regularity. Arch. Ration. Mech. Anal. 205(1), 195-237 (2012)

[16] Glass, O., Munnier, A., Sueur, F.: Point vortex dynamics as zero-radius limit of the motion of a rigid body in an irrotational fluid. Inventiones mathematicae 214(1), 171-287 (2018)

[17] Goudon, T., He, L., Moussa, A., Zhang, P.: The Navier-Stokes-Vlasov-Fokker-Planck system near equilibrium. SIAM J. Math. Anal. 42(5), 2177-2202 (2010)

[18] Goudon, T., Jabin, P.-E., Vasseur, A.: Hydrodynamic limit for the Vlasov-Navier-Stokes equations. Part I: light particles regime. Indiana Univ. Math. J. 53, 1495-1515 (2004)

[19] Goudon, T., Jabin, P.-E., Vasseur, A.: Hydrodynamic limit for the Vlasov-Navier-Stokes equations. Part II: fine particles regime. Indiana Univ. Math. J. 53, 1517-1536 (2004) 
[20] Hamdache, K.: Global existence and large time behaviour of solutions for the Vlasov-Stokes equations. Jpn. J. Ind. Appl. Math. 15(1), 51 (1998)

[21] Han-Kwan, D., Miot, É., Moussa, A., Moyano, I.: Uniqueness of the solution to the 2D Vlasov-Navier-Stokes system. Revista Matemática Iberoamericana 36(1), 37-60 (2020)

[22] Jabin, P.-E., Otto, F.: Identification of the dilute regime in particle sedimentation. Commun. Math. Phys. 250(2), 415-432 (2004)

[23] Kipnis, C., Landim, C.: Scaling Limits of Interacting Particle Systems, vol. 320. Springer, Berlin (2013)

[24] Oelschläger, K.: A law of large numbers for moderately interacting diffusion processes. Zeitschrift für Wahrscheinlichkeitstheorie und verwandte Gebiete 69(2), 279-322 (1985)

[25] Simon, J.: Compact sets in the spacel Lp(0, T;B). Annali di Matematica 146(1), 65-96 (1986)

[26] Sznitman, A.-S.: Topics in propagation of chaos. In: Ecole d'été de probabilités de Saint-Flour XIX-1989, pp. 165-251. Springer (1991)

[27] Yu, C.: Global weak solutions to the incompressible Navier-Stokes-Vlasov equations. Journal de Mathématiques Pures et Appliquées 100(2), 275-293 (2013)

Franco Flandoli

Scuola Normale Superiore of Pisa

Pisa

Italy

e-mail: franco.flandoli@sns.it
Cristiano Ricci

University of Pisa

Pisa

Italy

e-mail: cristiano.ricci@ec.unipi.it

Marta Leocata

Luiss Guido Carli

Roma

Italy

e-mail: mleocata@luiss.it

(accepted: February 24, 2021; published online: March 17, 2021) 